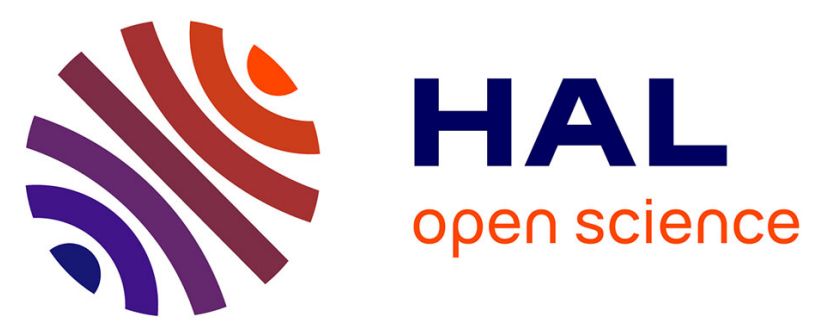

\title{
Damage growth modeling using the Thick Level Set (TLS) approach: Efficient discretization for quasi-static loadings
}

Paul-Emile Bernard, Nicolas Moës, Nicolas Chevaugeon

\section{- To cite this version:}

Paul-Emile Bernard, Nicolas Moës, Nicolas Chevaugeon. Damage growth modeling using the Thick Level Set (TLS) approach: Efficient discretization for quasi-static loadings. Computer Methods in Applied Mechanics and Engineering, 2012, 233, pp.11-27. 10.1016/j.cma.2012.02.020 . hal-01006715

\section{HAL Id: hal-01006715 https://hal.science/hal-01006715}

Submitted on 22 Jan 2018

HAL is a multi-disciplinary open access archive for the deposit and dissemination of scientific research documents, whether they are published or not. The documents may come from teaching and research institutions in France or abroad, or from public or private research centers.
L'archive ouverte pluridisciplinaire HAL, est destinée au dépôt et à la diffusion de documents scientifiques de niveau recherche, publiés ou non, émanant des établissements d'enseignement et de recherche français ou étrangers, des laboratoires publics ou privés. 


\title{
Damage growth modeling using the Thick Level Set (TLS) approach: Efficient discretization for quasi-static loadings
}

\author{
P.E. Bernard ${ }^{\mathrm{a}, \mathrm{b}, *}$, N. Moës ${ }^{\mathrm{b}}$, N. Chevaugeon ${ }^{\mathrm{b}}$ \\ ${ }^{a}$ Centre for Systems Engineering and Applied Mechanics, Université Catholique de Louvain, 4 Avenue Georges Lemaître, B-1348 Louvain-la-Neuve, Belgium \\ ${ }^{\mathrm{b}}$ GeM Institute, Ecole Centrale de Nantes, Université de Nantes, CNRS, 1 Rue de la Noë, F-44321 Nantes, France
}

\begin{abstract}
The Thick Level Set (TLS) model is a damage model containing a non-local treatment that prevents from spurious localization issues. It also offers an automatic transition from damage to fracture. The TLS approach to model damage growth was first presented in Möes et al. [24] with a first numerical imple-mentation for a time-dependent damage evolution law. In this paper, we propose some improvements in terms of discretization and explicit damage growth algorithms to obtain a robust, efficient and easy-to-implement model. These improvements include a simple and efficient variational formulation for computing the non-local quantities as well as the introduction of a so-called ramped Heaviside enrich-ment function to properly take into account the transition to cracks in fully damaged zones. We consider here a simple explicit formulation for quasi-static loadings, decoupling the elastic computation and the damage growth. The method is validated through standard benchmarks and compared to the Griffith's fracture theory. The convergence of energy and displacement errors is observed. More complex computations including damage initiations are eventually performed.
\end{abstract}

Keywords: Damage modeling, Thick Level Set, Extended finite element method, Fracture mechanics, Localization

\section{Introduction}

This work is motivated by the aim of building a model to predict the full scenario of degradation of quasi-brittle solids under quasistatic mechanical loading. Such a model involves the interaction of complex processes and obviously requires at least two main ingredients: fracture and damage mechanics.

On the one hand, although the basis of fracture mechanics can be considered as well-known, many open questions still remain about cracks modeling. For instance, processes as cracks merging or a single crack branching are still difficult to both model physically and represent numerically. Classical numerical representations of cracks, based on the extended finite element method [23] using level set functions (see e.g. [36,40,6,3]), quickly becomes a tedious task as the number of cracks grows. If cracks are not represented by level sets, merging may be handled with some care as in Budyn et al. [4] but this strategy will be most likely very cumbersome in three dimensions. Moreover, besides numerical issues, mechanical issues still remain with every single crack model. For instance, the cracks initiation, bifurcation and branching processes cannot be easily predicted. It is now commonly admitted that fracture mechanics alone is insufficient to model the entire

* Corresponding author at: Centre for Systems Engineering and Applied Mechanics, Université Catholique de Louvain, 4 Avenue Georges Lemaître, B-1348 Louvain-la-Neuve, Belgium.

E-mail address: paul-emile.bernard@uclouvain.be (P.E. Bernard). degradation process. Prediction of phenomena as crack initiation requires damage mechanics $[14,16]$. However, the link between damage and fracture is not obvious from both the physical and numerical point of view $[5,20,13,35]$.

Also, it is well known that local damage models may experience spurious localization and mesh dependencies when considering localization phenomena. Special care is thus required for the damage field treatment to avoid these problems. Several models have been developed to avoid these issues. All these models introduce somehow, explicitly or not, a characteristic length to obtain nonlocality. Classical non-local integral damage models are based on an averaging operator [2,29], integrating the local driving force on a given prescribed length, to obtain a non-local driving force, responsible for the damage growth. Such approaches may lead to relatively high computational costs. Indeed, averaging the local driving forces is done everywhere, even when no damaged zone is present yet. Moreover, the average itself requires to take topological interactions into account, especially when averaging close to notches, boundaries or cracks. The average around a point close to a crack lip for instance should not consider information from the other side of the crack (if considering an open crack). Solving these problematic topological interactions is not a priori trivial, especially when considering averaging operators based on a fixed characteristic length [27]. Another class of non-local models is the high-order gradient models. The main idea is to add a variable, as the damage gradient $[8,30,25]$ or the deformation gradient $[1,37,33]$, to the mechanical formulation. Second order operators 
then introduce the required diffusion to avoid spurious localizations. The additional cost of such methods cannot be neglected since new variables and corresponding relationship have been added to the problem over the whole domain, even where nonlocality is not required, not to mention the need to find appropriate boundary conditions for the unknown gradients. Hiding the nonlocality treatment into the formulation also makes it more difficult to control. Comparison between these two families of methods can be found in Peerlings et al. [27]. A third kind of method avoiding spurious localization is the phase-field approach $[10,9,15]$. A phase field varying from 0 to 1 is introduced to characterized the material degradation, providing a transition zone between sane and destroyed material. A new phase equation presenting a laplacian operator is added to the mechanical equations, providing somehow an additional characteristic length to the problem. This additional variational formulation is solved over the whole domain. Note finally that locating a crack in a highly damaged zone is a complex issue with all these classical non-local models.

The TLS model [24] has been developed to:

- Obtain an efficient and thermodynamically consistent non-local damage model.

- Provide answers to the fracture mechanics questions of crack initiation, branching and merging.

- Offer a natural transition between damage and fracture.

Let us stress the fact that the TLS model is a new theoretical damage model and not a pure numerical method. However, as level set based methods, it offers a natural and easy way to deal with topological crack-related issues.

The main idea of the TLS approach is to locate a damaged zone, possibly multiply connected, using a single level set function as depicted in Fig. 1. This easily solves the numerical questions of merging or branching. Indeed, level set functions, introduced in Osher and Sethian [26], have been widely used to represent material interfaces or free boundaries in an easy manner [22,18]. The isozero of the level set $\phi$ is called the damage front and corresponds to the transition between the sane and the damaged material. The level set $\phi$ considered is a signed distance function: its value $\phi(\boldsymbol{x})$ at point $\boldsymbol{x}$ is the minimum distance between $\boldsymbol{x}$ and the iso-zero of the level set field $\phi$. In the TLS model, damage is considered as a given (increasing) function of the level set, $d=d(\phi)$. As depicted in Fig. 1, the damage raises from 0 to 1 as $\phi$ goes from 0 to $l_{c}$ respectively. Therefore, the minimum distance between a point where $d=0$ and a point where $d=1$ is $l_{c}$, preventing spurious localization. It is important to notice, however, that the minimal distance between a point of the iso-zero and the iso- $l_{c}$ can be much larger than $l_{c}$ as illustrated in Fig. 1(between points A and B for instance).

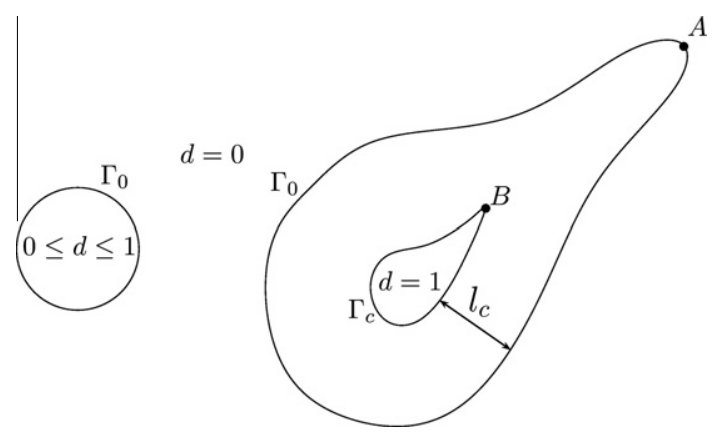

Fig. 1. A single level set function locating two damaged zones. The iso-zero $\Gamma_{0}$ locates the transition between the sane and damaged material. As the damage growth, the iso- $l_{c}$ appears, locating transition between damaged and fully damaged zones. This zone where $d=1$ can be seen as a macro crack.
As shown in Möes et al. [24], this approach is very promising because of the following reasons:

- The non-local treatment is restricted to damaged areas. It only requires a special treatment in the transition zone, where $0<d \leqslant 1$, leading to lower computational costs.

- The non-locality only appears where and when needed, without additional variable or equation to solve over the whole domain.

- The transition from damage to crack is automatic, no additional computation is required.

- The TLS model tends to the local model as the damage tends to zero: the damage initiation is simple and well-posed.

- Compared to standard non-local integral models, the characteristic length of the non-local operator is not fixed, but automatically varies from 0 to $l_{c}$ as the damage grows, avoiding topological issues around cracks.

- The approach is not a so-called "element deletion" method as in Saanouni et al.[32]: the fully damaged zone is delimited by the iso- $l_{c}$. Accurate integration in cut elements can be done while degrees of freedom are kept in the damage zone.

The objective of this paper is to present some improvements to the first TLS implementation given in Möes et al. [24], in particular to propose an efficient, simple and robust way to implement the TLS model, trivially extensible to three dimensions. Some fundamentals of the TLS damage model are recalled in Section 2. The reader is also referred to Möes et al. [24] for a detailed description of the theoretical model.

The paper is organized as follows: in Section 2, the governing equations are described including the damage evolution law. The local damage model is combined to the TLS to obtain the non-local model and some interesting properties of the TLS are stressed. Section 3 is devoted to the numerical resolution of the TLS model. We first introduce the ramped Heaviside enrichment function used in the elastic computation, with the aim to decouple degrees of freedom on both sides of the fully damaged zone. We insist at this point on the fact that this enrichment is not mandatory to use the TLS model, but provides a more accurate solution since it reduces the numerical dissipation induced along the macro-crack by a coarse mesh. A second paragraph is devoted to a variational formulation to efficiently compute the regularized damage energy release rate on the front using a standard finite element discretization. The last paragraph finally presents the explicit algorithm used to propagate the front as well as the level set update through a variational problem. The results are finally presented in Section 4: the TLS damage model is compared to Griffith's fracture theory in the case of the three points bending problem and errors are computed, leading to good convergence rates. Some standard benchmarks are then performed and the results are compared to experimental data. We finally illustrate the potential of the TLS approach by performing more complex computations where damage initiation plays an important role.

\section{Governing equations: from local to non-local models}

In this section, we first present the local governing equations. In order to simplify the TLS model equations, we choose to represent the damage in the simplest possible way using a single scalar damage variable. Indeed, in the framework of this paper, a scalar variable seems sufficient to present the main features of the model, its discretization, and to analyze its properties. Notice however that this is not a limitation of the TLS model. Anisotropic damage will for instance be considered in future works. The TLS model is introduced to derive the non-local damage evolution laws from the local behavior in Section 2.2. 


\subsection{A free energy involving dissymmetric behavior in tension/ compression}

We consider the domain of computation $\Omega$ bounded by $\Gamma$. The equilibrium equation reads:

$\nabla \cdot \boldsymbol{\sigma}=\mathbf{0}$ on $\Omega$

with $\sigma$ the Cauchy stress tensor and usual Neumann boundary conditions $\boldsymbol{\sigma} \cdot \boldsymbol{n}=\boldsymbol{f}$ with $\boldsymbol{f}$ the external loading on $\Gamma_{N}$ and $\boldsymbol{n}$ the outgoing normal vector to the domain boundaries. We consider the framework of small strains and displacements, providing the relation:

$\boldsymbol{\epsilon}=\frac{1}{2}\left(\nabla \boldsymbol{u}+(\nabla \boldsymbol{u})^{T}\right)$,

between the strain tensor $\boldsymbol{\epsilon}$ and the displacement field $\boldsymbol{u}$. Prescribed displacements $\boldsymbol{u}=\overline{\boldsymbol{u}}$ are imposed on $\Gamma_{D}$.

The stress is related to the strain through a state law derived from the free energy $\varphi$. We consider in the following a general expression for the free energy, $\varphi(\epsilon, d)$, yielding the state laws defined as the derivatives of the free energy:

$$
\left\{\begin{array}{l}
\boldsymbol{\sigma}=\frac{\partial \varphi}{\partial \epsilon}, \\
Y=-\frac{\partial \varphi}{\partial d} .
\end{array}\right.
$$

For instance, let us consider a simple scalar damage variable $d$ and the standard elastic free energy:

$\varphi(\boldsymbol{\epsilon}, d)=\frac{1}{2}(1-d) \boldsymbol{\epsilon}: \boldsymbol{E}: \boldsymbol{\epsilon}$,

with $\boldsymbol{E}$ the Hooke tensor. Using their definition (3), the state laws read:

$$
\left\{\begin{array}{l}
\boldsymbol{\sigma}=(1-d) \boldsymbol{E}: \boldsymbol{\epsilon} \\
Y=\frac{1}{2} \boldsymbol{\epsilon}: \boldsymbol{E}: \boldsymbol{\epsilon} .
\end{array}\right.
$$

We clearly observe here that the material presents the same behavior in tension and compression. In other words, the energy release rate $Y$ is independent of the signs of the strain eigenvalues and damage will grow even if the damaged zone only experiences compression. In some cases, this behavior may not be realistic and may lead to solutions drastically different from observations. Therefore, we use the following slightly non-linear free energy:

$\varphi(\boldsymbol{\epsilon}, d)=\mu\left(1-\alpha_{i} d\right) \epsilon_{i}^{2}+\frac{\lambda}{2}(1-\alpha d) \operatorname{tr}(\boldsymbol{\epsilon})^{2}$,

where $\lambda$ and $\mu$ are the Lamé elastic coefficients, $\epsilon_{i}$ the eigenvalues of the strain tensor and

$$
\begin{aligned}
\alpha_{i}=\beta & \text { if } \quad \epsilon_{i}<0, \\
1 & \text { if } \quad \epsilon_{i} \geqslant 0, \\
\alpha=\beta & \text { if } \operatorname{tr}(\epsilon)<0, \\
1 & \text { if } \operatorname{tr}(\epsilon \geqslant 0,
\end{aligned}
$$

with $0 \leqslant \beta \leqslant 1$. The case $\beta=1$ corresponds to the linear elastic potential (4). Indeed, the derivations with respect to $\epsilon$ and $d$ read:

$\left\{\begin{array}{l}\sigma_{i}=2 \mu(1-d) \epsilon_{i}+\lambda(1-d) \operatorname{tr}(\boldsymbol{\epsilon}), \\ Y=-\mu \operatorname{tr}\left(\boldsymbol{\epsilon}^{2}\right)-\frac{\lambda}{2} \operatorname{tr}(\boldsymbol{\epsilon})^{2},\end{array}\right.$

which is equivalent to (5).

On the contrary, if $\beta$ is set to zero, we obtain in complete tension:

$$
\left\{\begin{array}{l}
\sigma_{i}=2 \mu(1-d) \epsilon_{i}+\lambda(1-d) \operatorname{tr}(\boldsymbol{\epsilon}) \\
Y=-\mu \operatorname{tr}\left(\boldsymbol{\epsilon}^{2}\right)-\frac{\lambda}{2} \operatorname{tr}(\boldsymbol{\epsilon})^{2}
\end{array} \quad \text { if } \epsilon_{i} \geqslant 0 \quad \forall i\right.
$$

and in complete compression:

$$
\left\{\begin{array}{l}
\sigma_{i}=2 \mu \epsilon_{i}+\lambda \operatorname{tr}(\boldsymbol{\epsilon}) \\
Y=0
\end{array} \text { if } \epsilon_{i}<0 \quad \forall i\right.
$$

We thus observe the expected behavior: the damage has no effect on the material in compression. It recovers a complete stiffness in compression and the energy release rate becomes zero, meaning that damage does not grow. Note that the dissymmetric potential $\varphi$ is convex ${ }^{1}$ with respect to the strain for any damage value $(0 \leqslant d \leqslant 1)$. Other similar models involving closure effects may be found in Lemaitre and Desmorat [17].

Although the behavior of this dissymmetric potential is close to the well-known Mazars model [19], it is important to mention that it affects both the stress and the energy release rate state laws, providing thermodynamical consistency.

\subsection{Revisiting the damage evolution laws in the TLS model}

Let us now write the relationships of the local damage evolution and introduce the TLS representation to obtain the non-local quantities driving damage growth. The numerical computation of these quantities will be addressed on Section 3.2.

In this paper, we are interested by time-independent damage models. We consider the following well-known local behavior stating that damage grows as $Y$ reaches $Y_{c}$ :

$Y \leqslant Y_{c}, \quad \dot{d} \geqslant 0, \quad\left(Y-Y_{c}\right) \dot{d}=0$

Let us consider the case of the damaged zone depicted in Fig. 2. A portion $\delta s$ of the damage front $\Gamma_{0}$ moves of a distance $\delta \phi$. Because of the level set properties, this front advance has an impact on the whole zone sharing the same curvilinear coordinates $s$ (the gray zone in Fig. 2). The non-locality clearly appears here since the damage on a single point (say, for instance, point $P$ in Fig. 2) cannot grow without affecting the surrounding points sharing the same $s$ coordinate, preventing spurious localization. In the TLS framework, the damage is assumed to be a function of the level set, $d=d(\phi)$. The potential energy $E(\boldsymbol{u}, d)$ thus becomes a function of the level set:

$E(\boldsymbol{u}, \phi)=\int_{\Omega} \varphi(\boldsymbol{\epsilon}(\boldsymbol{u}), d(\phi)) d \Omega-\int_{\Gamma_{N}} \boldsymbol{f} \cdot \boldsymbol{u} d \Gamma$,

with $\boldsymbol{f}=\boldsymbol{\sigma} \cdot \boldsymbol{n}$ the imposed load on $\Gamma_{N}$. This relation can be differentiated using the relationship:

$\delta \varphi=\frac{\partial \varphi}{\partial \epsilon} \delta \epsilon+\frac{\partial \varphi}{\partial d} \delta d$,

the state laws (3) and the integration by part of the equilibrium (1) on $\Omega$. The amount of dissipated energy as the front moves of a distance $\delta \phi(s)$ on a front part $\delta s$ then reads:

$\delta E=-\int_{\Omega} Y d^{\prime} \delta \phi(s) d \Omega$.

This quantity, the front energy release rate, is the required information to predict the damage variation. One may observe here a huge advantage of the TLS model: the damage derivative $d^{\prime}(\phi)$ is zero everywhere except in the damaged zone. Therefore, computing this variation of energy only requires integration in the damaged zone $\Omega^{d}$. The treatment of the non-locality is thus "local", unlike classical non-local models which require important numerical efforts even in zones where $d=0$. The computational cost required for propagating the damage then becomes clearly much smaller.

Explaining the TLS approach requires now to introduce a change of variables. ${ }^{2}$ Let us rewrite the dissipated energy (12) in the orthogonal basis $(\phi, s)$ depicted in Fig. 2 using the variable change $d \Omega=\left(1-\frac{\phi}{\rho(s)}\right) d \phi d s$ as previously done in Möes et al. [24]:

\footnotetext{
1 This is not necessarily true for all similar potentials, for instance the similar potential $\varphi(\boldsymbol{\sigma}, d)$ written as a function of the stress eigenvalues.

2 Note that the following are theoretical observations, of course not required for the actual finite element computation.
} 

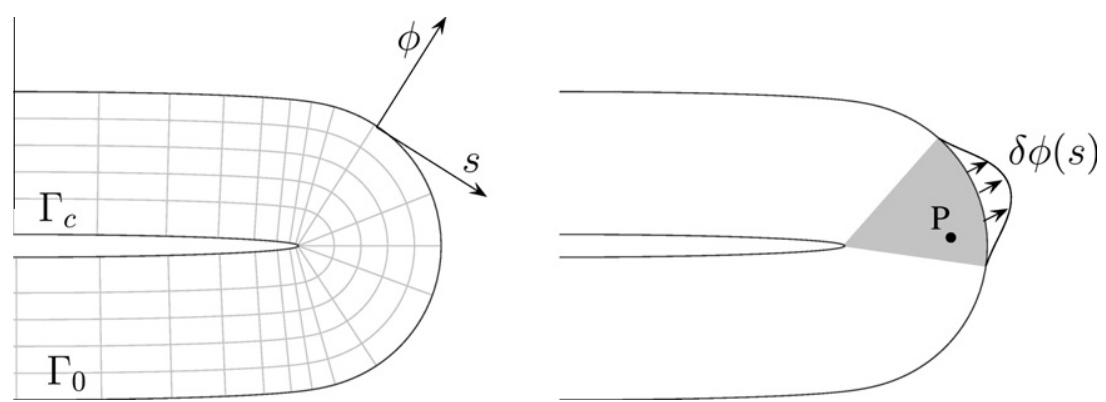

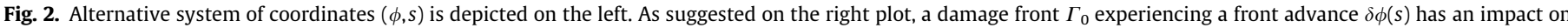

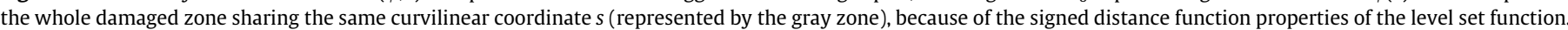

$\delta E=-\int_{\Gamma_{0}} \underbrace{\int_{0}^{l} Y(\phi, s) d^{\prime}(\phi)\left(1-\frac{\phi}{\rho(s)}\right) d \phi}_{g(s)} \delta \phi(s) d s$,

with $l$ comprised between 0 (damage initiation) and at most $l_{c}$ (leading to a fully damaged zone) and $\rho$ being the front curvature on $\Gamma_{0}$. We see here appearing a quantity of dimension $\operatorname{dim}(\Omega)-1$ defined on $\Gamma_{0}$, the configurational force $g(s)$. Once $Y$ homogenized on the band width $\phi \in[0, l]$, the front propagation is thus basically a problem of dimension $\operatorname{dim}(\Omega)-1$.

It is now possible to fulfill the homogenized version of the local propagation law (10) by searching $g=g_{c}$ :

$\int_{\Gamma_{0}} \int_{0}^{l} Y(\phi, s) d^{\prime}(\phi)\left(1-\frac{\phi}{\rho(s)}\right) d \phi \delta \phi(s) d s$

$$
=\int_{\Gamma_{0}} \int_{0}^{l} Y_{c}(\phi, s) d^{\prime}(\phi)\left(1-\frac{\phi}{\rho(s)}\right) d \phi \delta \phi(s) d s,
$$

for all front advance $\delta \phi$.

However, $g(s)$ and $g_{c}(s)$ clearly tend to 0 as $l \rightarrow 0$. Therefore, finding the load factor required to obtain a front advance leads to comparing two quantities tending to zero. Hence, unlike the first TLS paper [24], we choose another quantity, $\bar{Y}(s)$, defined as a constant value along $\phi$ such that:

$g(s)=\int_{0}^{l} Y(\phi, s) d^{\prime}(\phi)\left(1-\frac{\phi}{\rho(s)}\right) d \phi=\int_{0}^{l} \bar{Y}(s) d^{\prime}(\phi)\left(1-\frac{\phi}{\rho(s)}\right) d \phi$.

Since $\bar{Y}$ depends only on $s$, it can be removed from the integral to obtain its alternative definition:

$\bar{Y}(s)=\frac{\int_{0}^{l} Y(\phi, s) d^{\prime}(\phi)\left(1-\frac{\phi}{\rho(s)}\right) d \phi}{\int_{0}^{l} d^{\prime}(\phi)\left(1-\frac{\phi}{\rho(s)}\right) d \phi}$.

This homogenized quantity $\bar{Y}$ is thus a simple weighted average of $Y$, tending to $Y$ as $l$ tends to zero. The homogenized propagation condition finally simply reads:

$\int_{\Gamma_{0}} \bar{Y}(s) \delta \phi d s=\int_{\Gamma_{0}} \overline{Y_{c}}(s) \delta \phi d s \quad \forall \delta \phi(s)$,

with $\overline{Y_{c}}$ the same weighted average of $Y_{c}$. If $Y_{c}$ is uniform over the domain, $\overline{Y_{c}}=Y_{c}$. We observe here that the TLS model is also wellposed in the sense that the limit of the defect tending to zero (damage initiation) corresponds to the local model, $Y=Y_{c}$, since:

$\lim _{l \rightarrow 0} \bar{Y}=Y$.

Non-locality thus only appears for $d>0$. Finally, the front evolution related to the local damage evolution (10) reads:

$\bar{Y} \leqslant \overline{Y_{c}}, \quad \dot{a} \geqslant 0, \quad\left(\bar{Y}-\overline{Y_{c}}\right) \dot{a}=0$.
If more complex evolution laws than (10) are considered, the strategy introduced in Möes et al. [24] may be used to obtain the front evolution relating $\bar{Y}$ and $\dot{a}$.

\section{Variational formulations and discretization}

In this work, we use a simple explicit algorithm, decoupling the elastic computation and the damage front propagation. We first consider the damage as a given field and compute elastic fields. The first paragraph is devoted to this first step and to the introduction of a new enrichment function. Then, based on these elastic fields, we compute the damage front propagation. A robust and efficient variational formulation is presented in the second paragraph to compute the non-local variables $\bar{Y}$ and $\overline{Y_{c}}$ in the damaged zone. The third paragraph is devoted to the numerical computation of the time-independent model and to the actual damage propagation.

\subsection{Elastic computation using ramped Heaviside enrichment}

The standard Galerkin formulation is obtained by multiplying Eq. (1) by a smooth function $\hat{\boldsymbol{u}} \in \mathcal{U}_{0}$ and integrating on the domain, with $\mathcal{U}_{0}$ the appropriate function space of admissible test displacements:

$\mathcal{U}_{0}=\left\{v: v \in H^{1}(\Omega), \quad v=0\right.$ on $\left.\Gamma_{D}\right\}$.

Integrating the divergence by parts, the weak formulation reads:

$\int_{\Omega} \boldsymbol{\sigma}: \boldsymbol{\epsilon}(\hat{\boldsymbol{u}}) d \Omega=\int_{\Gamma_{N}} \boldsymbol{f} \cdot \hat{\boldsymbol{u}} d \Gamma \quad \forall \hat{\boldsymbol{u}} \in \mathcal{U}_{0}$.

The displacement solution belongs to the space of admissible trial displacements:

$\mathcal{U}=\left\{v: v \in H^{1}(\Omega), \quad v=\bar{u}\right.$ on $\left.\Gamma_{D}\right\}$.

We seek an approximation $\boldsymbol{u}^{h}$ of $\boldsymbol{u}$ such that $\boldsymbol{u}^{h} \in \mathcal{U}_{p}^{h}$ with $\mathcal{U}_{p}^{h} \subset \mathcal{U}$ the finite dimensional space of admissible trial displacements composed of polynomials $N^{p}$ of degree at most $p$ :

$\boldsymbol{u}^{h}=\sum_{j=1}^{\mathcal{N}} N_{j}^{p} \boldsymbol{U}_{j} \quad \in \mathcal{U}_{p}^{h}$

where $\boldsymbol{U}_{i}$ are the nodal values and $\mathcal{N}$ is the number of degrees of freedom.

The Newton-Raphson loop on the iterate $\boldsymbol{u}_{i}^{h}$ to solve the nonlinear potential $(6)$ reads:

$$
\begin{aligned}
\int_{\Omega} \boldsymbol{\epsilon}\left(\Delta \boldsymbol{u}^{h}\right): \boldsymbol{H}: \boldsymbol{\epsilon}\left(\hat{\boldsymbol{u}}^{h}\right) d \Omega= & \int_{\Gamma_{N}} \boldsymbol{f} \cdot \hat{\boldsymbol{u}}^{h} d \Gamma \\
& -\int_{\Omega} \boldsymbol{\sigma}\left(\boldsymbol{u}_{i}^{h}\right): \boldsymbol{\epsilon}\left(\hat{\boldsymbol{u}}^{h}\right) d \Omega \quad \forall \hat{\boldsymbol{u}}^{h} \in \mathcal{U}_{0_{p}^{h}},
\end{aligned}
$$


with $\Delta \boldsymbol{u}^{h}=\boldsymbol{u}_{i+1}^{h}-\boldsymbol{u}_{i}^{h}$ and $\boldsymbol{H}$ the tangent operator. Details about its computation can be found in the appendix. Note that the tangent operator can be singular in the case of a complete damage in tension. In this case, a residual stiffness is imposed to the tangent operator to prevent singularities, but the right hand side is computed exactly. We therefore converge to a solution satisfying the equilibrium in spite of this approximation in the tangent operator.

To obtain a proper solution, we still need to introduce some enrichment in this formulation. Indeed, let us consider the fully damaged zone depicted in Fig. 3. The strain field on the left was computed without any enrichment. The consequence is that, since using continuous finite elements, the degrees of freedom on both sides of the completely damaged zone are still connected. Nodes in the fully damaged zone should be properly enriched to decouple both sides of the crack, providing tension-free crack lips. A node in the zone $d=1$ must be enriched if it presents a support (i.e. the set of surrounding elements) cut twice (or more) by the iso- $l_{c}$, as node " $n$ " in Fig. 3. On the other hand, enrichment is not required if the support is cut only once as for instance the nodes of element " $e$ " in Fig. 3.
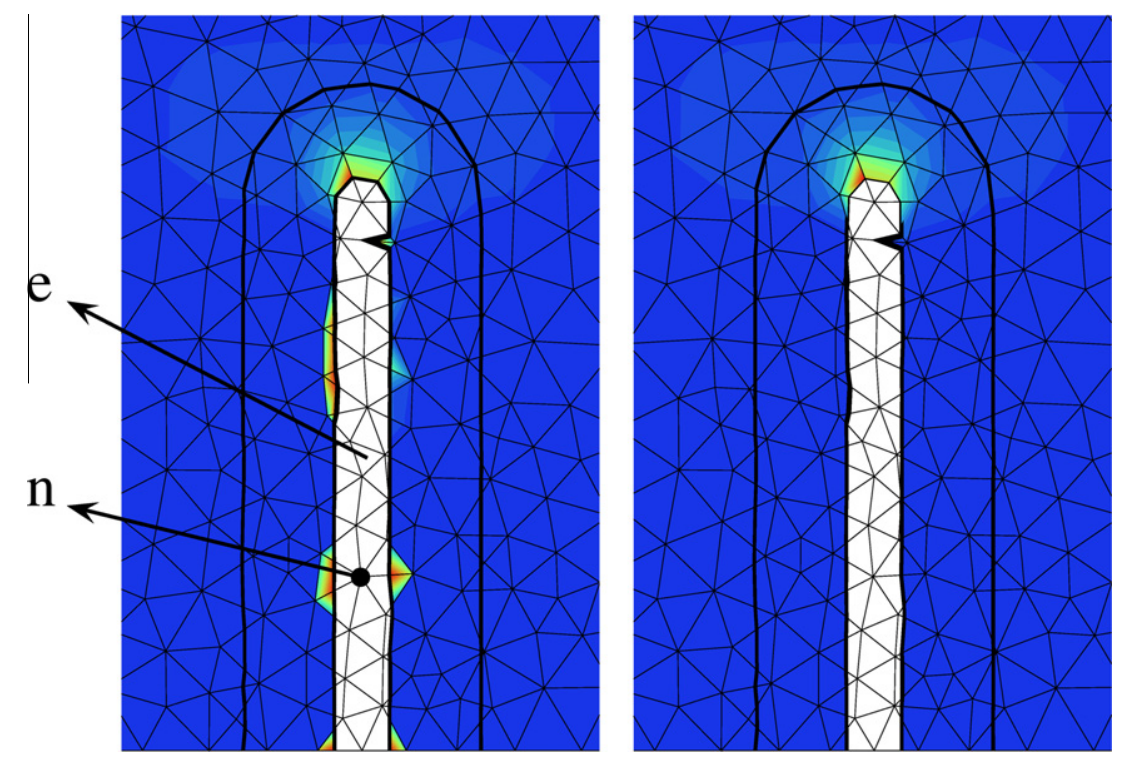

Fig. 3. Close-up view of the strain field on the three points bending specimen. Without enrichment (left), crack lips experience high strain at several spots and both sides of the fully damaged zone (white zone) are still coupled. With the use of the ramped Heaviside enrichment (right), we obtain a complete decoupling in tension, yielding to tension-free crack lips, mandatory for an accurate computation of the damage propagation.
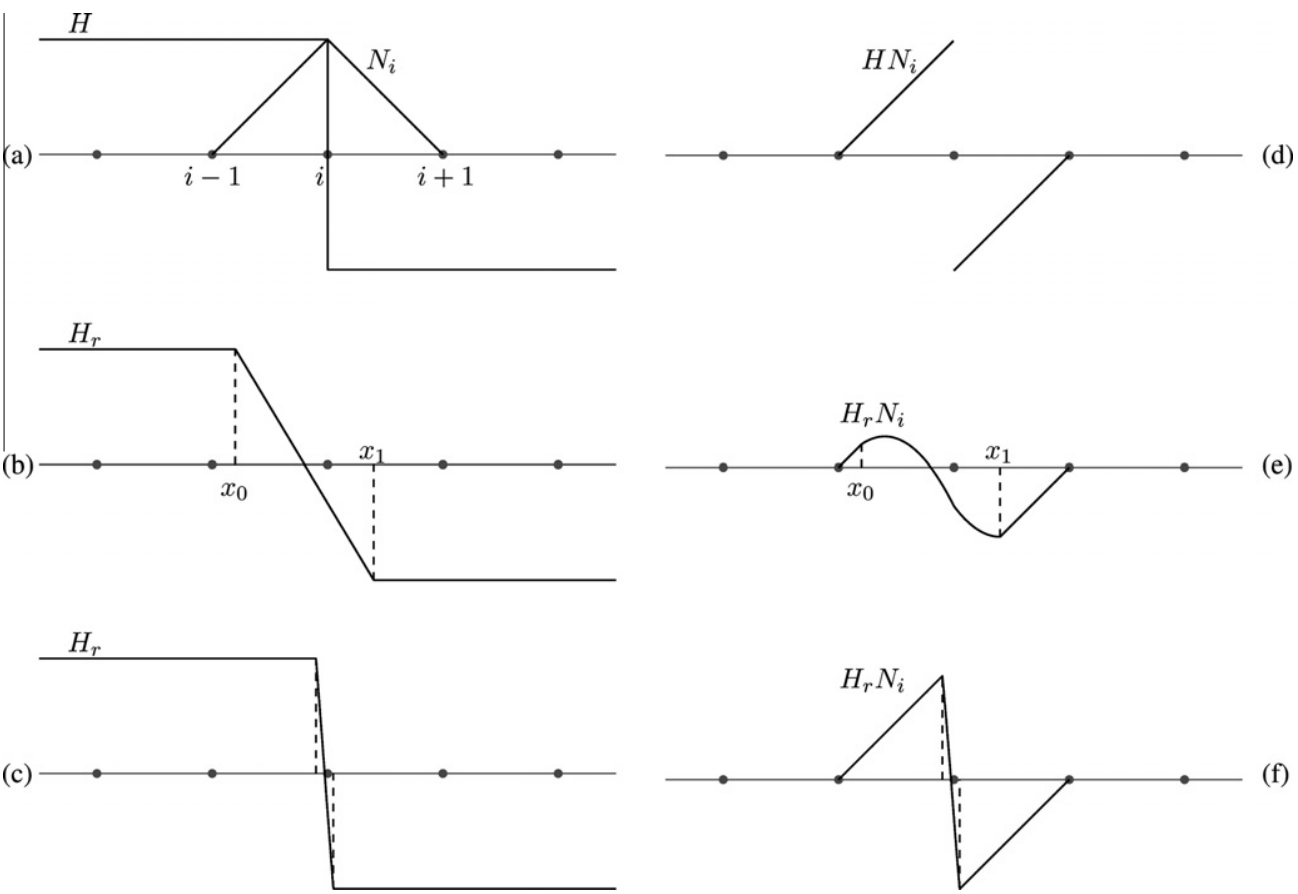

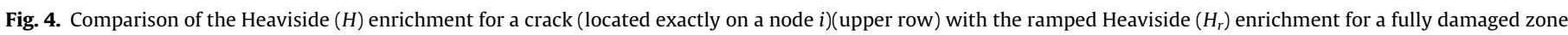

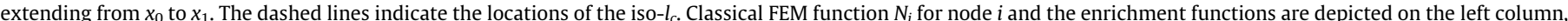

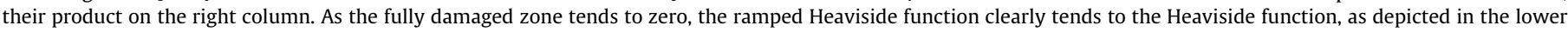
row. 
In the extended finite element method, both sides of a crack are decoupled by enriching the mesh with a Heaviside enrichment [23]. To handle the case depicted in Fig. 3, we introduce an enrichment being +1 on one side of the fully damaged zone and -1 on the other side. Then, we connect +1 and -1 values by a continuous ramp in the fully damaged area. If the material law is dissymmetric as in (6), contact will be automatically handled since the material in the fully damaged zone will recover its stiffness under compression. We shall call this enrichment ramped Heaviside.

Let us illustrate this on the one-dimensional example depicted in Fig. 4, with a crack located exactly on node $i$ (Fig. 4(a)). Using classical X-FEM method for a single crack, the product of the shape function $N_{i}$ and the Heaviside function $H, H N_{i}$ (Fig. 4(d)), is the classical additional discontinuous function required in the finite element basis to represent a discontinuous field on node $i$. In the following, we consider that the level set function is discretized as a piecewise linear function. Therefore, the elements are cut only once by the iso- $l_{c}$, and we can consider that there is always at least one node in the fully damaged zone. Let us assume the fully damage zone delimited by $x \in\left[x_{0}, x_{1}\right]$ in Fig. $4(\mathrm{~b})$. We thus define the ramped Heaviside function as:

$$
H_{r}(x)= \begin{cases}1 & \text { if } x \leqslant x_{0} \\ 1-\frac{2}{x_{1}-x_{0}}\left(x-x_{0}\right) & \text { if } \quad x_{0}<x<x_{1} . \\ -1 & \text { if } x \geqslant x_{1}\end{cases}
$$

As the fully damaged zone tends to zero, as illustrated in Fig. 4(c), the additional function $H_{r} N_{i}$ clearly tends to the classical $H N_{i}$. Although the ramped Heaviside function is continuous, it yields to a complete decoupling of the two sides of the fully damaged zones since the stiffness in this zone is zero for tension loading. But once associated to a dissymmetric potential resisting in compression, its continuity also ensures that the contact between the two sides is satisfied. Contact is therefore automatically handled with this approach.

In practice, for two- or three-dimensional applications, building a ramped Heaviside function with the definition (22) can be very

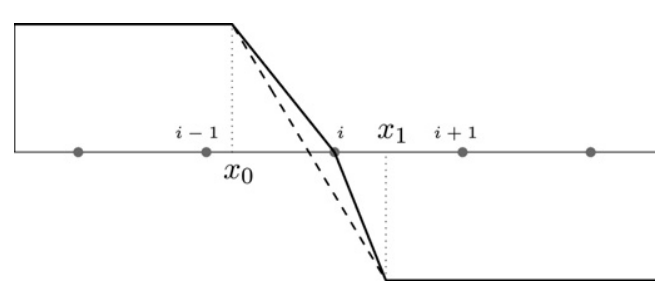

Fig. 5. In practice, the ramped Heaviside is actually much easier to build using piecewise linear functions defined on every element in the damaged zone (solid line) instead of one single linear function (dashed line). complicated. A much more simple approach consists in considering that the function is zero at node $i$, i.e. the nodes in the fully damaged zone, as illustrated in Fig. 5.

Let us consider now a two-dimensional application to illustrate the potential of this approach. The domain and computational mesh is depicted in Fig. 6, where the white zones correspond to a complete damage $d=1$ (complete loss of stiffness in tension) and the gray zones to an undamaged stiffness. We first apply appropriate Dirichlet rigid translation conditions on each block of this domain (see Fig. 6), avoiding contact between blocks, using the linear elastic potential (4). We obtain motion of perfect rigid bodies (upper row in Fig. 7), each block being completely independent of the others and experiencing a zero strain field. Without applying any boundary condition, the global stiffness matrix presents 33 null eigenvalues, corresponding to the rigid modes of the 11 independent blocks. On the other hand, when applying a constant pressure on the four boundaries using potential (6), the material recovers its stiffness (lower row in Fig. 7) and the contact is naturally handled on the blocks interfaces. This demonstrates the capability of the ramped Heaviside enrichment to numerically take into account the dissymmetric behavior of the material.

\subsection{Variational formulation for the non-local model}

We now wish the front to move when the homogenized quantity $\bar{Y}$ reaches $\overline{Y_{c}}$. Let us recall its definition (15):

$\int_{0}^{l} \bar{Y}(s) d^{\prime}(\phi)\left(1-\frac{\phi}{\rho(s)}\right) d \phi=\int_{0}^{l} Y(\phi, s) d^{\prime}(\phi)\left(1-\frac{\phi}{\rho(s)}\right) d \phi$.

First, we would like to avoid direct computation of the Jacobian depending on the curvature $\rho$. Integrating this relation along the damage front to obtain a variational formulation leads to integrals on $\Omega^{d}$ : the curvature computation is therefore not required. The major issue for the numerical discretization is the fact that this quantity $\bar{Y}(s)$ is clearly of a dimension $\operatorname{dim}(\Omega)-1$, along the damage front. This problem can be written as the following variational formulation: find $\bar{Y} \in \overline{\mathcal{Y}}$ such that:

$\int_{\Omega^{d}} \bar{Y} \widehat{\bar{Y}} d^{\prime}(\phi) d \Omega=\int_{\Omega^{d}} Y \widehat{\bar{Y}} d^{\prime}(\phi) d \Omega \quad \forall \hat{\bar{Y}} \in \overline{\mathcal{Y}}$,

with the constrained space:

$\overline{\mathcal{Y}}=\left\{y\right.$ "regular" on $\Omega^{d}: y_{, \phi}=\nabla y \cdot \nabla \phi=0$, i.e. $\left.y(\phi, s)=y(s)\right\}$,

strongly imposing the fact that $\bar{Y}$ is constant along the level set gradient, i.e. only function of the curvilinear coordinate $s$. The formulation is very simple, but the difficulty lies here in the numerical construction of such a constrained space. One possible way to implement this is to parametrize the front curvature $s$, and extend this parametrization into the damaged zone, as done in Möes
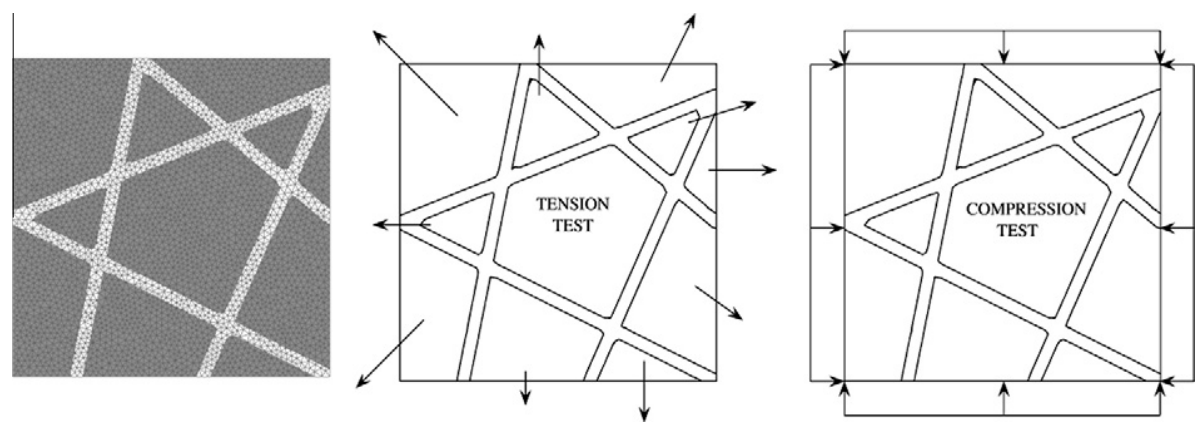

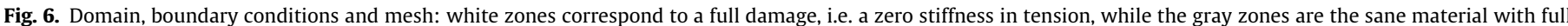

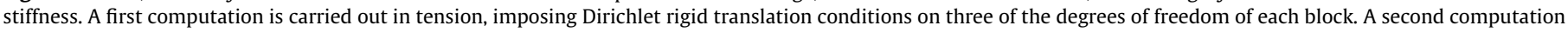
considers a constant pressure on all boundaries. 


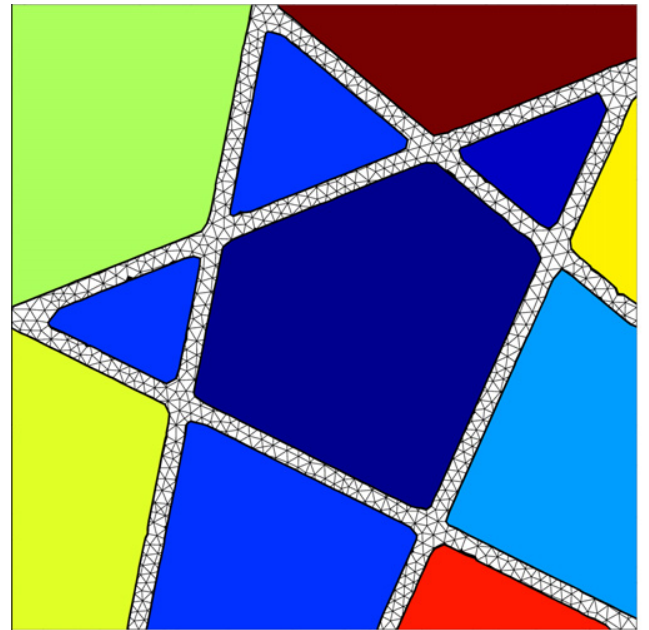

displacement

$1.25 \mathrm{e}-05 \quad 2.51 \mathrm{e}-05$

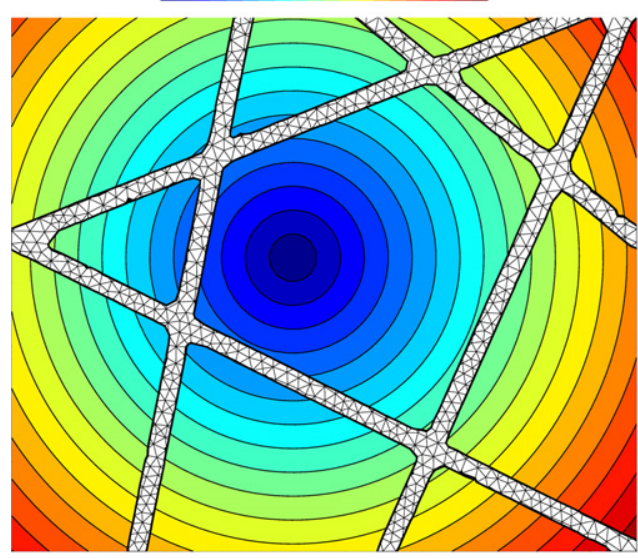

displacement

0

0.764

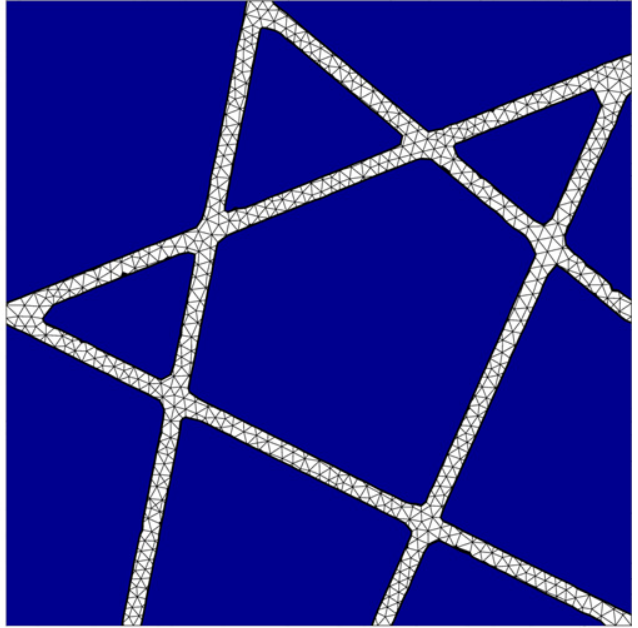

strain
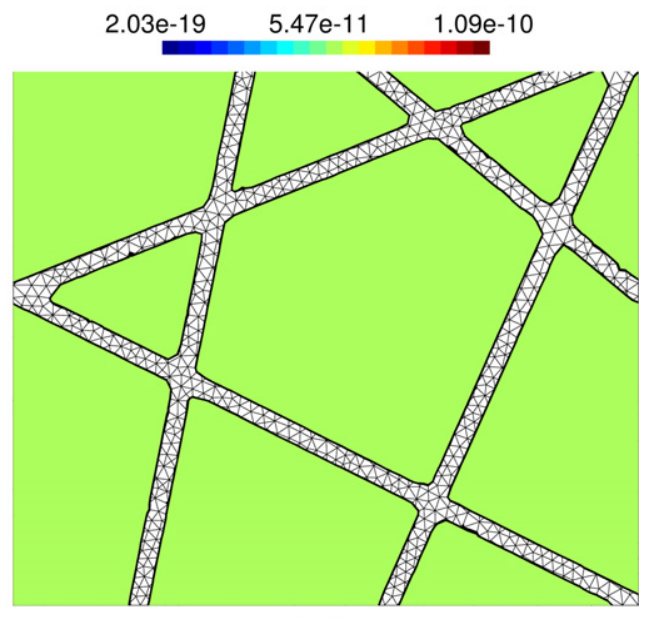

strain

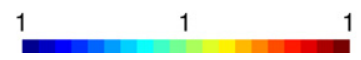

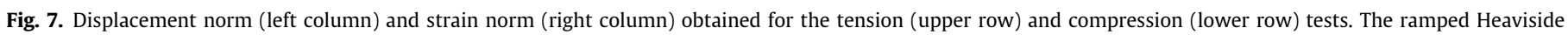
enrichment allows the different sane blocks to experience rigid body motion in tension, while the degraded zone is able to recover stiffness in compression.

et al. [24]. The field $\bar{Y}$ is therefore easily discretized. But it requires a parametrization of the front, which is not trivial if $\operatorname{dim}(\Omega)=3$, or in presence of multiple fronts using parallel computing.

We therefore choose another approach to weakly impose the fact that $\bar{Y}$ is constant along the level set gradient, working directly on the variational formulation itself instead of using a complicated space. In other words, we consider in the discretization that $\bar{Y}=\bar{Y}(\phi, s)$ instead of $\bar{Y}(s)$, and weakly impose the constraint $\nabla \bar{Y} \cdot \nabla \phi=0$. Let us define the following simple spaces $\mathcal{Y}$ and $\mathcal{Y}_{0}$ as:

$\mathcal{Y}=\left\{y\right.$ "regular" on $\left.\Omega^{d}\right\}$

$\mathcal{Y}_{0}=\left\{y\right.$ "regular" on $\Omega^{d}$ and $y=0$ on $\left.\Gamma_{0}\right\}$.

Finding $\bar{Y}$ can be achieved by considering the following variational formulation:

Find $\bar{Y} \in \mathcal{Y}, \lambda \in \mathcal{Y}_{0}$ such that:

$$
\left\{\begin{array}{l}
\int_{\Omega^{d}}\left(\bar{Y} d^{\prime}(\phi) \hat{\bar{Y}}+\frac{h^{2}}{l_{c}} \nabla \bar{Y} \cdot \nabla \widehat{\bar{Y}}\right) d \Omega \\
\quad+\int_{\Omega^{d}} l_{c}(\nabla \lambda \cdot \nabla \phi)(\nabla \overline{\bar{Y}} \cdot \nabla \phi) d \Omega=\int_{\Omega^{d}} Y d^{\prime} \hat{\bar{Y}} d \Omega \quad \forall \hat{\bar{Y}} \in \mathcal{Y}, \\
\int_{\Omega^{d}} l_{c}(\nabla \hat{\lambda} \cdot \nabla \phi)(\nabla \bar{Y} \cdot \nabla \phi) d \Omega=0 \quad \forall \hat{\lambda} \in \mathcal{Y}_{0}
\end{array}\right.
$$

where $(\nabla \lambda \cdot \nabla \phi)$ is a Lagrange multiplier to enforce the condition $\nabla \bar{Y} \cdot \nabla \phi=0$ and $h$ denotes the characteristic element size. The diffusion term in $h^{2}$ has been introduced to get rid of possible small oscillations on $\bar{Y}$. Note that this term is not necessary to ensure the stability of the formulation.

We then simply discretize $\bar{Y}$ and $\lambda$ in the same way, in the support of the damaged zone, as a sum of piecewise linear functions:

$$
\begin{aligned}
\bar{Y}^{h} & =\sum_{i} L_{i} \bar{Y}_{i}, \\
\lambda^{h} & =\sum_{i} L_{i} \lambda_{i},
\end{aligned}
$$

with $L_{i}$ the standard linear Lagrange shape functions. Finally, the Dirichlet boundary conditions on $\lambda$, see (24), is approximated by setting $\lambda$ to zero at all nodes of the elements crossed by $\Gamma_{0}$.

The computation of $\bar{Y}$ is therefore eventually very simple and robust using formulation (25). It only requires a standard finite element space defined only in the damaged zone, leading to solving a small system made of mass and diffusive matrices, trivially extended to a three-dimensional configuration. If the critical energy release rate $Y_{c}$ is not a constant value, the exact same system is solved to compute its homogenization $\overline{Y_{c}}$, to be compared to $\bar{Y}$. 


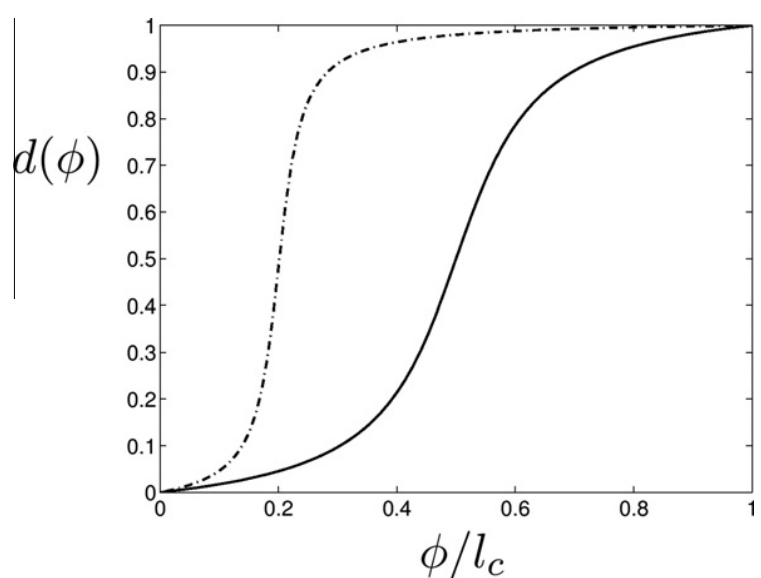

Fig. 8. Damage shape $d(\phi)$ used in all computations. The dashed-dotted profile corresponds to the $L$-shaped panel while other computations are performed using the solid profile.

\subsection{Damage front propagation and explicit algorithm for the time- independent model}

Based on a given level set configuration (denoted by the lower script $n$ ) $\phi_{n}$, we computed the elastic problem for a reference load, say $\widetilde{F}$, and found the damage front energy $\widetilde{Y}_{n}(s)$ (the upper bar is omitted to simplify the notations) using the variational formulation (25). The discretization of $\bar{Y}$ on the front is known as a sum of modes with nodal values $\bar{Y}_{i}$. We now wish to find a simple explicit algorithm to propagate the damage front according to the homogenized time-independent local law, $\bar{Y}=\overline{Y_{c}}$. We also wish to find the required load $F$, or the required load factor $\mu=F / \widetilde{F}$, to obtain this front advance. We consider here the general case where $Y_{c}$ is not constant, i.e. $\overline{Y_{c}}$ can experience variations along $\Gamma_{0}$. In order to capture possible snap-backs, a generalization of the crack length control is used: a norm of the front advance is imposed between two time steps. This is taken to be the infinite norm (maximum front advance) in this paper.

First, we want at least one of the modes $\bar{Y}_{i}$ to be "activated", i.e. leading to a front advance. For a given damage configuration and due to the state laws used in this paper, the displacement (and strain) is proportional to the load factor $\mu$. The energy release rate is therefore proportional to its square, $\bar{Y}=\mu^{2} \widetilde{Y}$, and the smallest load factor required to obtain a front advance is obviously given by

$\mu_{n}^{2}=\min _{i}\left(\frac{\overline{Y_{c i}}}{\widetilde{Y}_{i}}\right)$.

For a constant value of $Y_{c}$, this corresponds to the largest value of $\widetilde{Y}$. In the framework of an explicit formulation, we impose that this mode will lead to a front advance of $a_{\max }$, typically the size of an element.

Now, the main question still remains: are other modes activated, which means other front parts moving, and what is their front advance $a_{i}$ ? Indeed, assume that we only move the front part corresponding to the maximum mode. Then, we obtain the new level set $\phi_{n+1}$, leading to a new load factor $\mu_{n+1}^{2}$. For such an explicit load increment, $\Delta \mu_{n}^{n+1}=\mu_{n+1}-\mu_{n}$, maybe other modes should have been activated in the configuration at time step $n$. In an implicit formulation, we would have to compute the sensitivity of $\bar{Y}$ to the front advance $a$. One could also choose to iterate between time steps $n$ and $n+1$ to optimize the front position. However, we choose here a simpler explicit approach. At time step $n$, we assume the following relationship between the front advance $a_{n}=\phi$ ${ }_{n}+1-\phi_{n}$ and the front energy $\bar{Y}_{n}$ : $a_{i n}=k\left\langle\left(\mu_{n}+|\Delta \mu|\right)^{2}\left(\frac{\widetilde{Y}_{i}}{\overline{Y_{c i}}}\right)_{n}-1\right\rangle$,

with values:

$\Delta \mu=\Delta \mu_{n-1}^{n}$,

$k=\frac{a_{\max }}{\left(1+\frac{\left|\Delta \mu_{n-1}^{n}\right|}{\mu_{n}}\right)^{2}-1}$,

where $a_{\max }$ is the maximum front advance allowed at each time step, i.e. the "crack length" control parameter. This value of parameter $k$ is chosen to obtain consistency between time steps. More details about these parameters can be found in the appendix.

Finally, updating the level set still requires to know the level set increment $a$ anywhere in the domain and not just on the front. We thus wish to extend the information from the front along the level set gradient, to obtain $\phi_{n+1}=\phi_{n}+a$ everywhere in the domain. There are many possible ways to extend the information from the front to the whole domain, see for instance Sethian [34]. We choose here to solve the following problem: find $a \in \mathcal{A}$ such that:

$\int_{\Omega}(\nabla a \cdot \nabla \phi)(\nabla \hat{a} \cdot \nabla \phi) d \Omega=0 \quad \forall \hat{a} \in \mathcal{A}_{0}$.

This variational formulation, imposing the condition $\nabla a \cdot \nabla \phi=0$ in the domain, simply corresponds to a diffusion operator in the level set gradient direction. The test and trial admissible spaces are respectively defined as:

$$
\begin{aligned}
& \mathcal{A}_{0}=\left\{a: a \in H^{1}(\Omega), a=0 \text { on } \Gamma_{0}\right\}, \\
& \mathcal{A}=\left\{a: a \in H^{1}(\Omega), a=\bar{a} \text { on } \Gamma_{0}\right\} .
\end{aligned}
$$

The level set increments $a$ are known on the nodes surrounding the front $\Gamma_{0}$ and are thus imposed as Dirichlet boundary conditions.

Note finally that, as many level set-based numerical methods, the TLS method relies on the distance function properties of the level set in the damaged zone. Therefore, it is mandatory to guarantee that the level set remains a signed distance function though some "re-initialization" process [34].

\subsection{Damage initiation}

As stated in the previous section, the non-local model tends to the local one as the defect size tends to zero. Indeed, the average of the energy release rate $\bar{Y}$ clearly tends to $Y$ in this case. In other words, the criterion for damage initiation is local and reads $Y=Y_{c}$. Afterwards, non-locality gradually steps in as damage grows. Again, we choose to use an explicit algorithm as simple as possible: after each elastic computation on a front configuration $\phi_{n}$, we seek for the maximum of $Y_{n}$ (based on the loading factor $\left.\mu_{n-1}\right)$ in the whole domain. When this maximum is larger than $Y_{c}$, we initiate a circular defect with an approximate radius of $h$, i.e. the smallest defect that can be represented on our mesh. Note

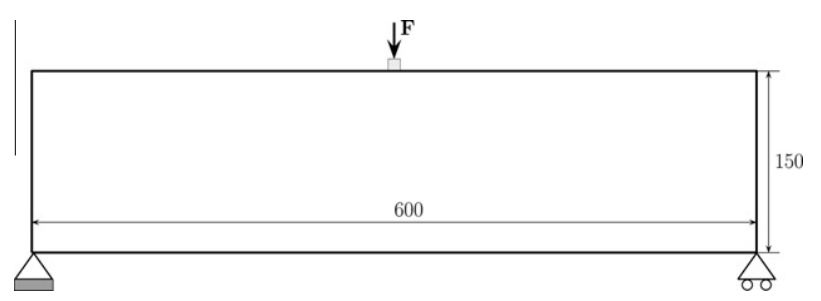

Fig. 9. Domain definition and boundary conditions for the three points bending problem, with dimensions in millimeters. We consider a beam thickness of $0.1 \mathrm{~m}$ without initial notch. 
that with $h \ll l_{c}$, the maximum damage in this initiated defect is also much smaller than 1 . This damage initiation is thus of course much less invasive than initiating a crack and has a negligible impact on the global stiffness of the structure.

\section{Results}

The first paragraph concerns a simple benchmark, the three points bending problem, to quantitatively validate the non-local

Table 1

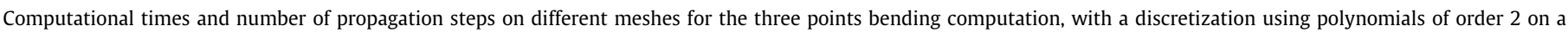
single Intel Xeon Processor at $2.00 \mathrm{GHz}$.

\begin{tabular}{|c|c|c|c|c|c|c|}
\hline Element size & 0.0465 & 0.0345 & 0.0240 & 0.0169 & 0.0120 & 0.0085 \\
\hline Triangles & 1194 & 2210 & 4420 & 8754 & 17188 & 34478 \\
\hline Propagation steps & 187 & 265 & 368 & 528 & 795 & 1204 \\
\hline Time (min) & 7 & 22 & 67 & 216 & 780 & 3163 \\
\hline
\end{tabular}

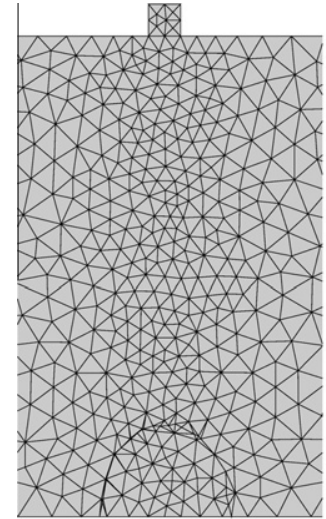

time step 36

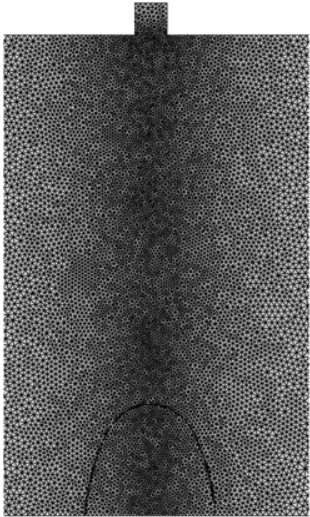

time step 280

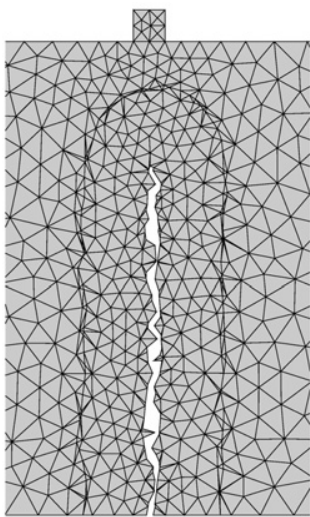

time step 164

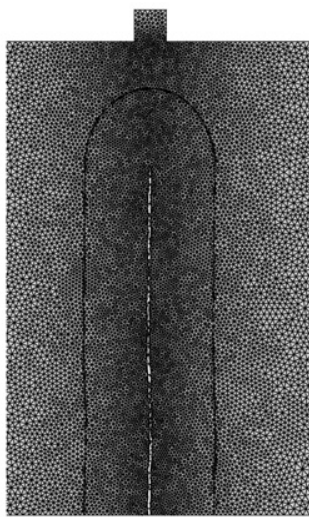

time step 1064

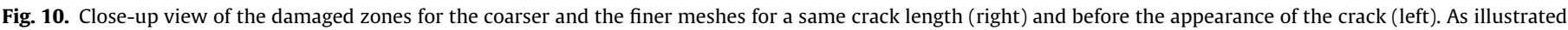

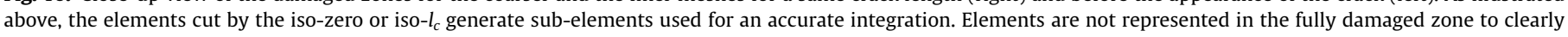
visualize the macro-crack.
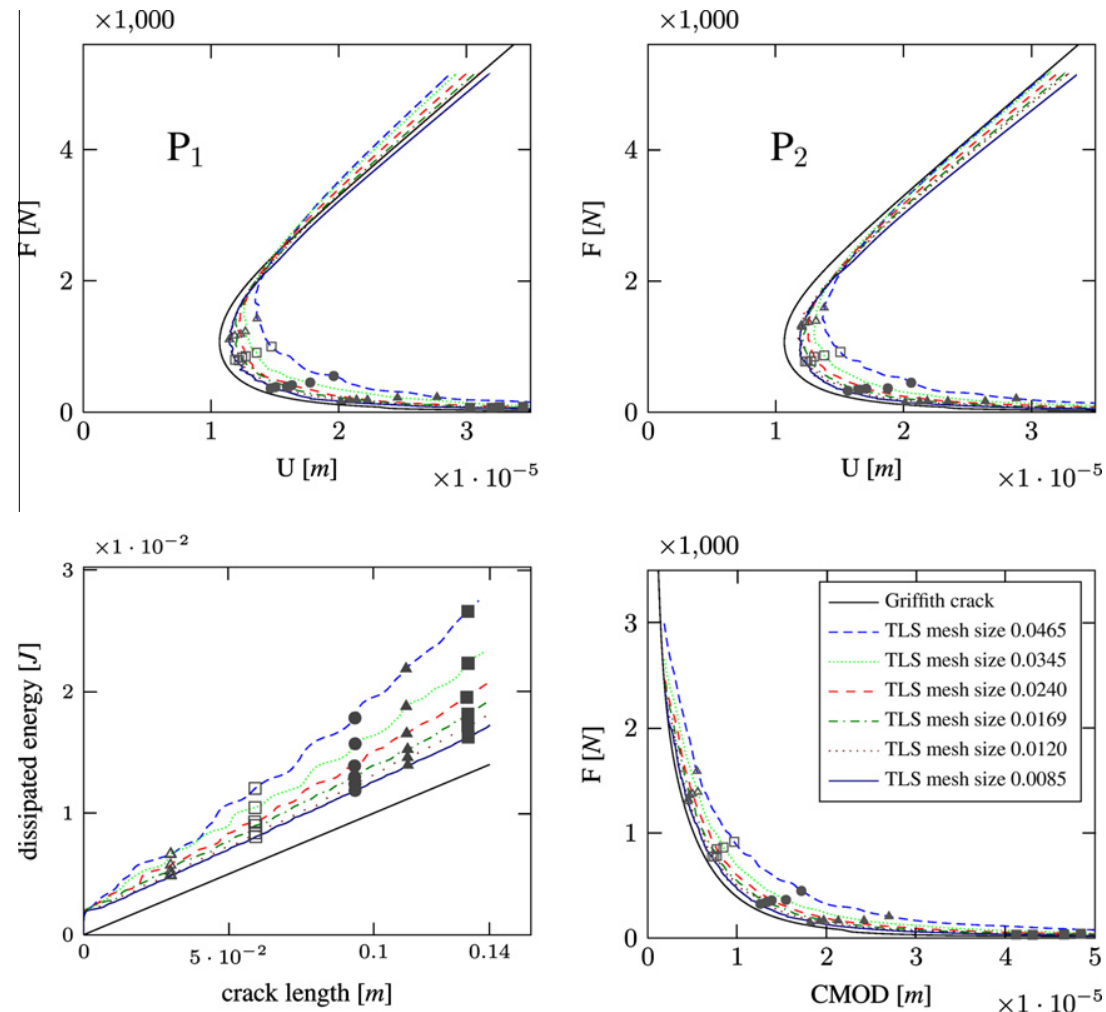

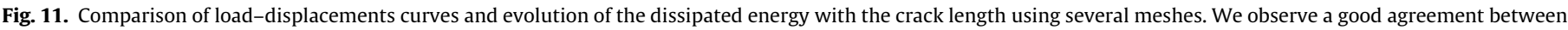

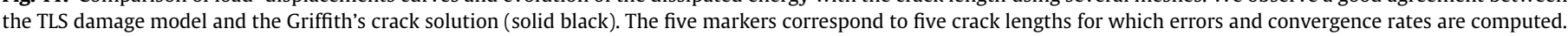


model by comparison with Griffith's theory and computation of error convergence rates. A second benchmark is the L-shaped panel, presenting a mixed tension-compression fracture. Both the crack path and the load-displacement curves are compared with experimental data. Other benchmarks are used to illustrate the potential of the TLS model, and in particular the damage initiation process. Although the check for possible damage initiation is done in all computations, multiple initiation only occurs in the computation of the Brazilian test as in the benchmark of a multiply perforated plate in tension.

Let us summarize the only three parameters used in the following TLS computations. The first one is of course the shape of the damage with respect to the level set. We use in the following computations the arc-tangent profile depicted in Fig. 8:
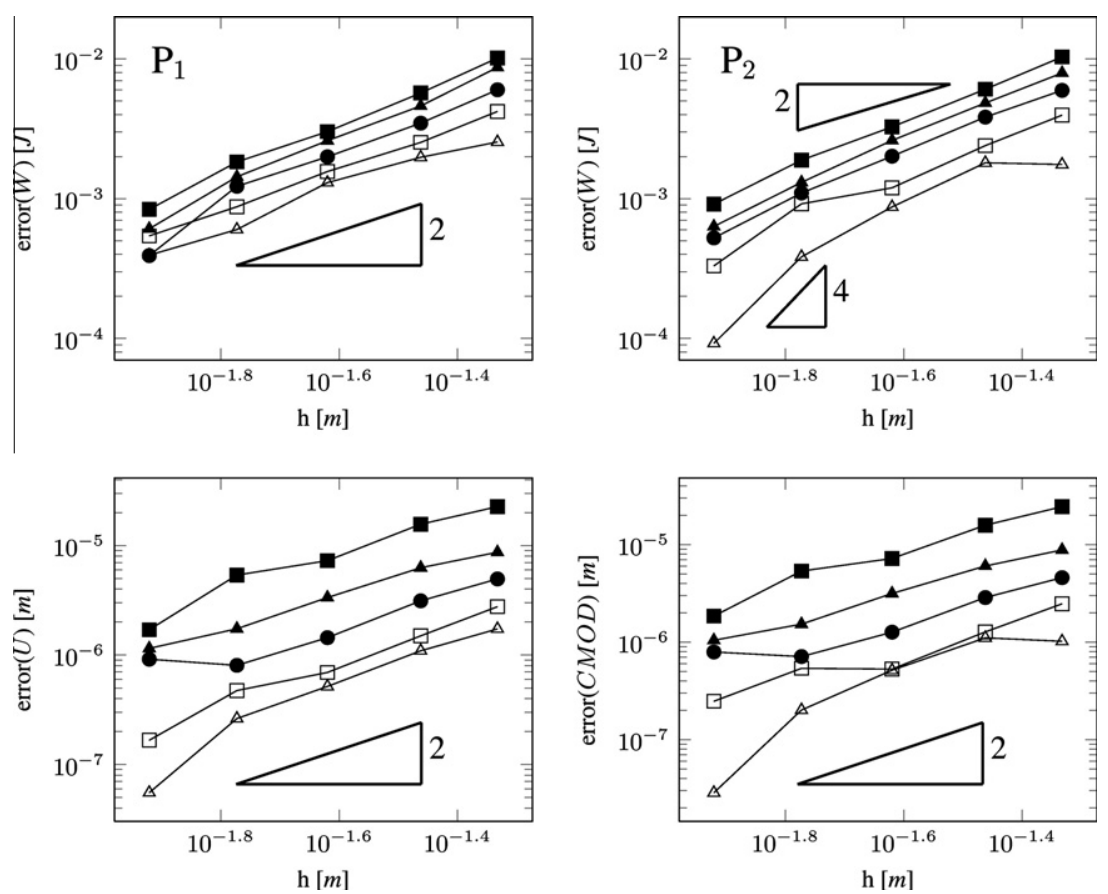

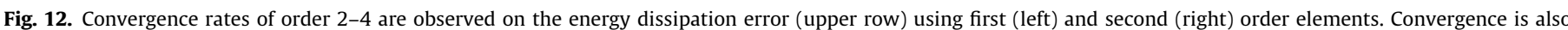
observed on the displacement and CMOD errors (bottom row). The five markers correspond to the five crack lengths on Fig. 11.
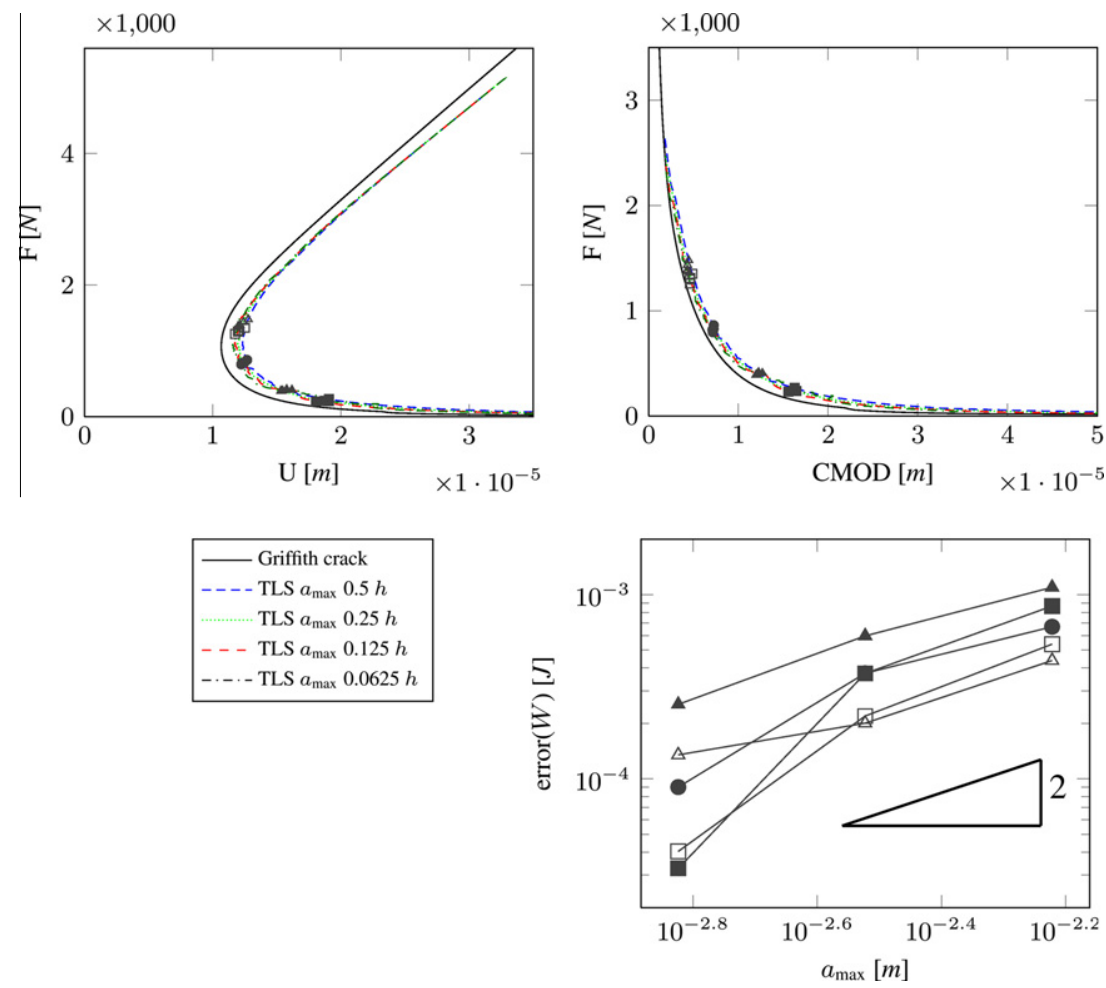

Fig. 13. Using the explicit algorithm on a given mesh, convergence is observed in $a_{\max }$, i.e. the maximum level set growth at each iteration. 

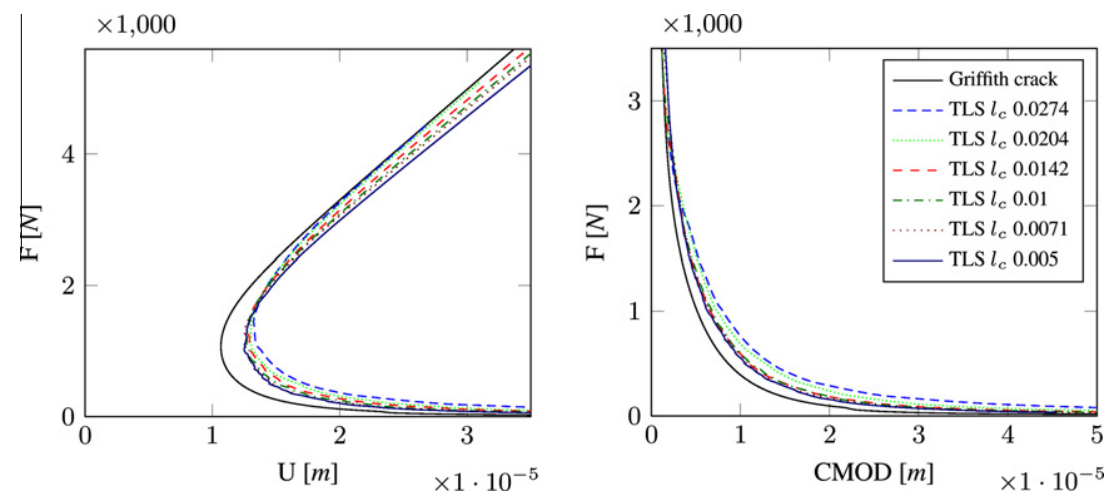

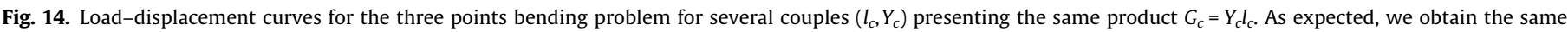

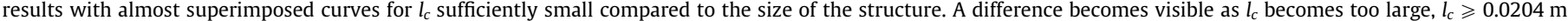
(about $13 \%$ of the height of the beam).

$d(\phi)=c_{2} \operatorname{atan}\left(c_{1}\left(\frac{\phi}{l_{c}}-c_{3}\right)\right)+c_{4}$

Most of the computations are performed with $c_{1}=10$ and $c_{3}=0.5$ (solid line on Fig. 8), except the L-shaped panel where $c_{1}=35$ and $c_{3}=0.2$ (dashed-dotted line on Fig. 8). The constants $c_{2}$ and $c_{4}$ are determined to satisfy the conditions $d\left(l_{c}\right)=1$ and $d(0)=0$ respectively:

$$
\left\{\begin{array}{l}
c_{2}=\left(\operatorname{atan}\left(c_{1}\left(1-c_{3}\right)\right)-\operatorname{atan}\left(-c_{1} c_{3}\right)\right)^{-1}, \\
c_{4}=-c_{2} \operatorname{atan}\left(-c_{1} c_{3}\right) .
\end{array}\right.
$$

The two remaining parameters are the characteristic length $l_{c}$ and $Y_{c}$. One way to choose these values is to compare the TLS model to fracture mechanics. Considering a crack in an infinite domain, i.e. with the shape of the equivalent damage front remaining unchanged, it has been shown in Möes et al. [24] that the fracture energy $G_{c}=2 A Y_{c} l_{c}$, with A the area under the curve $d(\phi)$. Therefore, every couple $\left(Y_{c}, l_{c}\right)$ presenting the same product will lead to the same energy dissipation and the same load-displacement curve in the well established crack propagation regime.

Unless specified otherwise, all computations were performed using second order polynomial finite elements to discretize the displacement field. For all computations, the initiated damaged zones have a size of the order of $h$, the element size. The plane strain assumption is considered in all test cases.

\subsection{The three points bending problem: comparison with Griffith's fracture theory}

The geometry and boundary conditions are depicted in Fig. 9. The TLS damage model is quantitatively compared to the Griffith's fracture theory, the latter being solved using the standard X-FEM method. The Griffith computation is initiated with a crack of $1.5 \mathrm{~mm}$. The errors on relevant quantities as the energy dissipation or displacements are then computed using several meshes with decreasing characteristic element sizes and the convergence rates are computed.

As shown in Möes et al. [24], when $l_{c}$ is small compared to the size of the structure, results of the Griffith's fracture and the TLS damage theories should be close to each other. The Young modulus and Poisson ratio used are respectively $E=36.5 \mathrm{GPa}$ and $v=0.1$, while we choose a critical fracture energy $G_{c}=1 \mathrm{Nm}^{-1}$ for the Griffith's fracture computation. The propagation direction of the crack is imposed in the crack solution, while it is of course determined by the TLS model in the TLS computations.

First, computations are performed on several meshes presenting different element sizes (see Table 1), the Griffith solution being computed on the finer mesh. The finer and coarser meshes are depicted in Fig. 10. The maximum front propagation distance used here is $a_{\max }=h / 8$ with $h$ the element size. The values $l_{c}=0.02 \mathrm{~m}$ and $Y_{C}=50 \mathrm{~Pa}$ have been chosen to obtain the same fracture energy $G_{c}=1 \mathrm{Nm}^{-1}$.

In Fig. 10 are depicted the damage transition zones on the coarser and finer mesh, for a same crack length. The crack length is defined here as the maximum vertical coordinate of the iso- $l_{c}$. The damage front position is similar while the fully damaged zone width is of about one element, i.e. the minimum size to activate the ramped Heaviside enrichment.

We may observe the efficiency of the TLS model in terms of computational costs on the computational times reported in Table 1. The computational cost of the non-local damage treatment and propagation represents about $10 \%$ of the elastic computational cost.

In Fig. 11 are the resulting load-displacement curves. We visually observe a convergence of the curves with the spatial discretization and a good agreement with Griffith's solution. On the evolution of the dissipated energy with the crack length, we

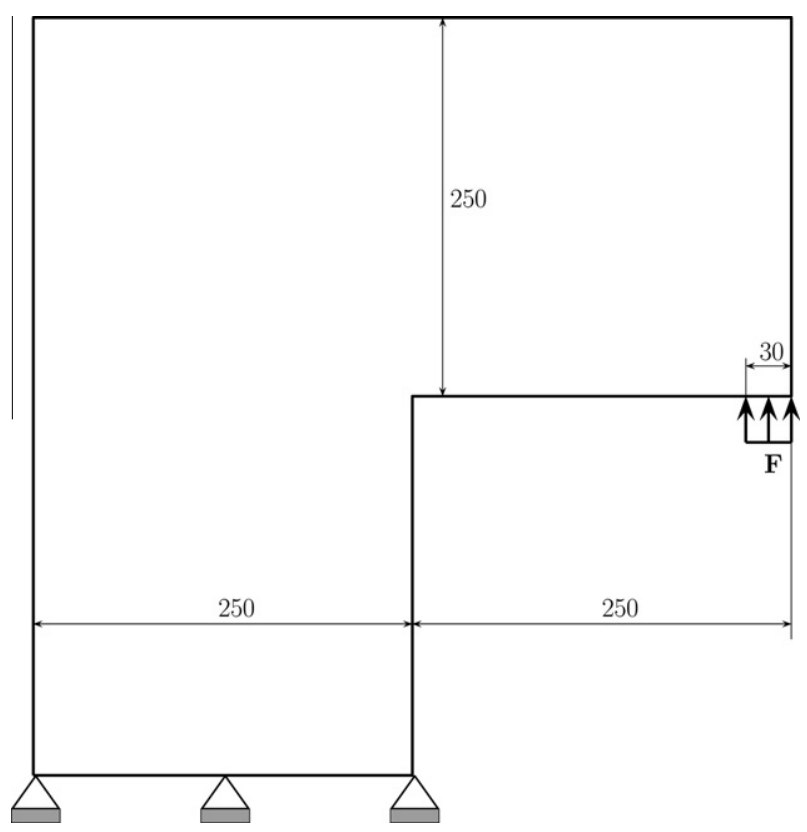

Fig. 15. Domain definition and boundary conditions for the $L$-shaped panel test, with dimensions in millimeters. 

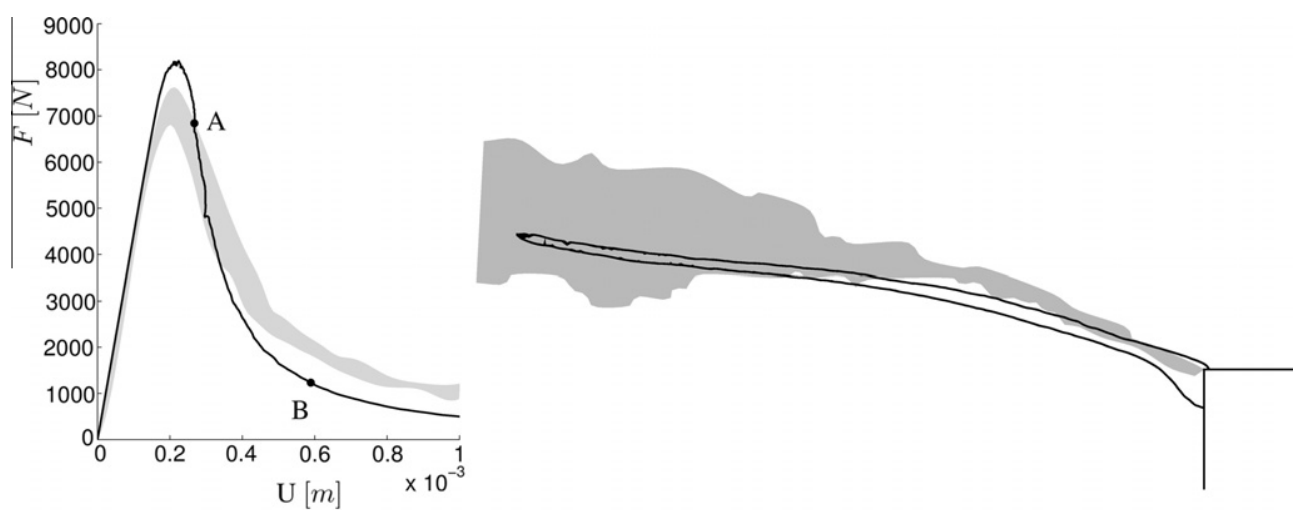

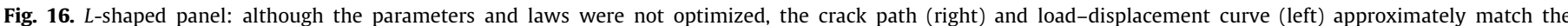
experimental measurements (gray envelopes).

(a)

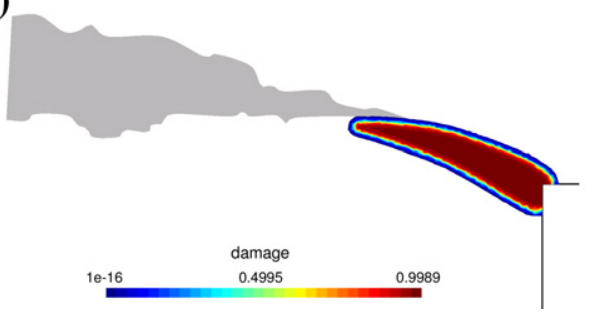

(b)

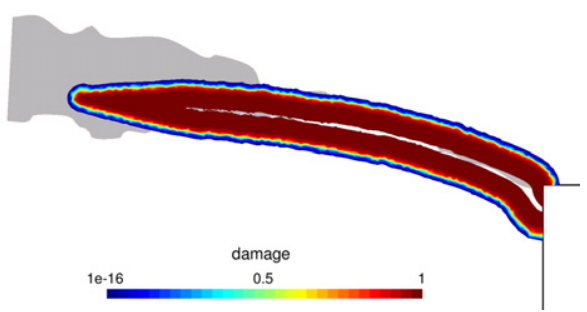

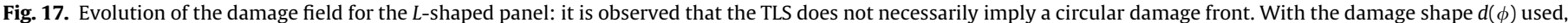

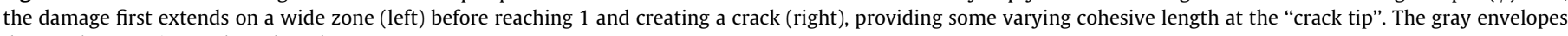
denote the experimental crack path.

observe that the slopes of the TLS curves tend to the Griffith's slope, which is consistent with the equivalency $G_{c}=2 A Y_{c} l_{c}$. However, some energy is also dissipated before the crack appears. This amount of energy clearly depends on the length $l_{c}$ and the TLS and Griffith's curves thus merge for $l_{c} \rightarrow 0$. Five crack lengths have been chosen, represented by the five different markers in Fig. 11, to compute errors and their corresponding convergence rates. The errors on the dissipated energy $(W)$, the displacement under the load $(U)$ and the crack mouth opening displacement (CMOD) are computed using the finer mesh solution as reference. Errors are reported in Fig. 12. First, we clearly observe a convergence, with a rate of at least 2, using polynomials of order 1 or 2 . Using polynomials of order 2 , the convergence rate seems to be of order 4 for the small crack length (triangle markers with the smallest absolute error), and then tends to 2 for larger crack lengths. This can be explained by the fact that we use a simple explicit algorithm to propagate the damage front, with a maximum advance of the front of $h / 8$. Therefore, the error in pseudo-time as no reason to converge as fast as the spatial error and will finally alter the global convergence rate for high-order polynomials. Note also that we try to compare values for a same crack length. But since the time steps are different, the crack lengths are not exactly the same, which might also introduce a significant error.

A second experiment consists in using, on the same fine mesh, different maximum values for the front propagation. We observe in Fig. 13 the convergence of the explicit propagation algorithm with $a_{\max }$ using the four values $a_{\max }=h / 2, h / 4, h / 8$ and $h / 16$. Convergence rate of 2 with the explicit time step is obtained, as expected.

Finally, in Fig. 14 are depicted the results for different couples $\left(Y_{c}, l_{c}\right)$ having the same product $G_{c}$. Different meshes have been used for these computations, presenting the same ratio $h / l_{c}$ to ensure that the driving fields inside the damage band are discretized with the same accuracy. The curves should be theoretically the same (in an infinite domain) since they present the same dissipation. However, the domain is finite. We observe the expected result on Fig. 14. The curves are almost superimposed, which enlightens the fact that, for $l_{c}$ sufficiently small compared to the characteristic size of the structure, the product $Y_{c} l_{c}$ is much more important than the single value of $l_{c}$.

\subsection{The L-shaped panel}

Specimen dimensions and boundary conditions are depicted in Fig. 15. This benchmark, elaborated by Winkler [39], provides

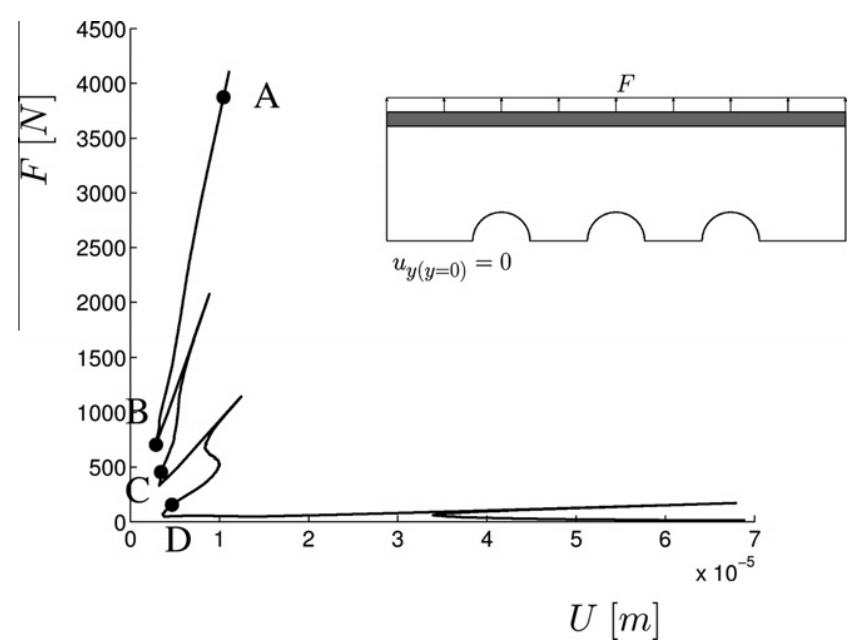

Fig. 18. Load-displacement curve and domain definition and boundary conditions for the three holes plate. 
experimental data to compare with and involves a mixed tensioncompression curved cracking. Several studies previously used this benchmark, as the study of cohesive and cohesionless cracks models [21], the comparison of crack path tracking strategies [12], the development of X-FEM-based models for cohesive cracks [38] or the study of crack models comparison using adaptive grids [7].

Unlike the other TLS computations, we used different parameters for the arc-tangent profile leading to the dashed-dotted profile of Fig. 8 to obtain a better agreement with the experimental data. The load-displacement curve is depicted in Fig. 16 as well as the crack path. The experimental results are represented by the gray envelopes on Figs. 16 and 17.

The main difference with the other computations done in this paper is the importance of the initiation stage, and its impact on the load-displacement curve. Indeed, we can observe the large impact of the damage profile $d(\phi)$ during the initiation on the evolution of the damage field on Fig. 17. Using this modified arc-tangent profile leads to the creation of a large zone where the damage does not reach 1 , until the step denoted " $A$ ". Then, this kind of "cohesive" zone keeps moving with the crack tip (i.e. the zone $d=1$ ), but its length becomes smaller and finally tends to $l_{c}$ as the damage front reaches the left edge of the panel. The shape of the damage profile has thus a large impact on the load-displacement curve and on the cohesive behavior of the damaged zone.

Although parameters were not really optimized to match the experiment, we observe a relatively good agreement on the crack path and the load-displacement response, even without using any hardening behavior or cohesive traction-separation interface law.

\subsection{Damage initiation in a multiply perforated plate}

We consider here a plate presenting three holes. Loading and boundary conditions are represented in Fig. 18, taking into account

(a)

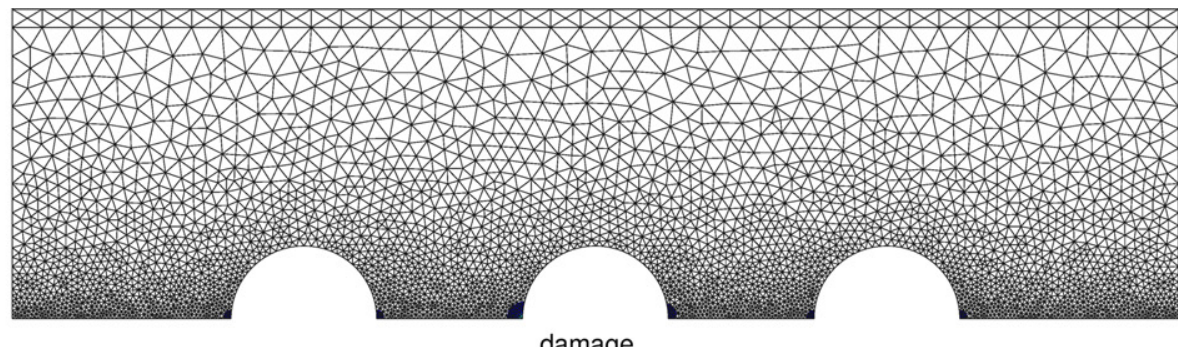

damage

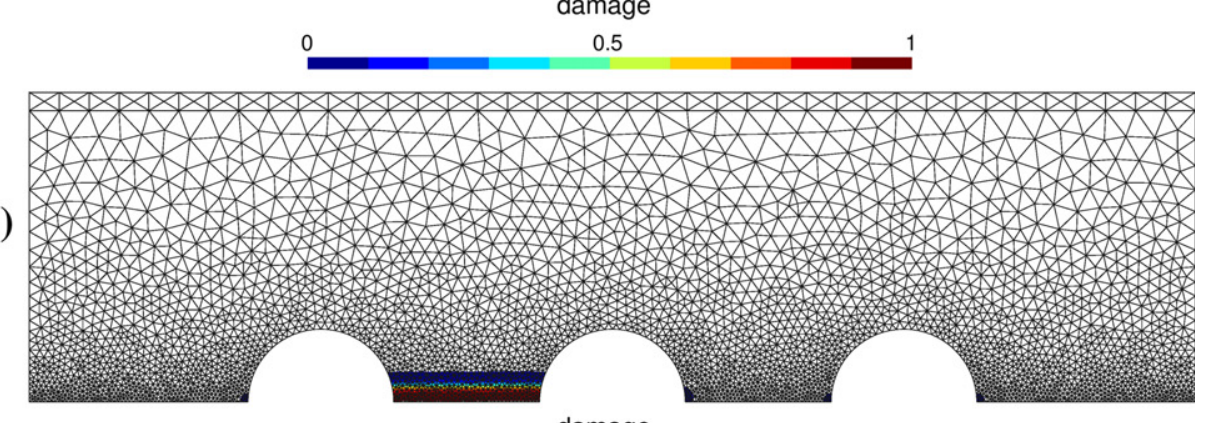

damage

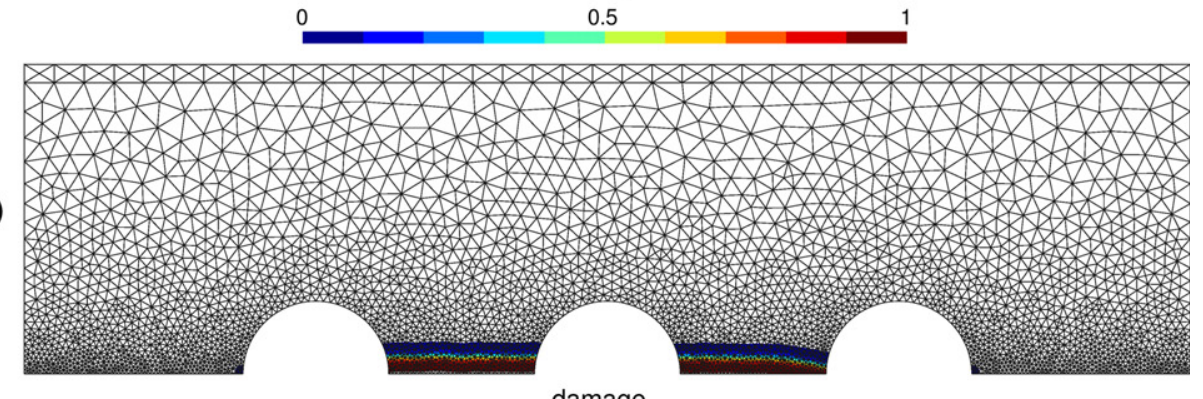

amage

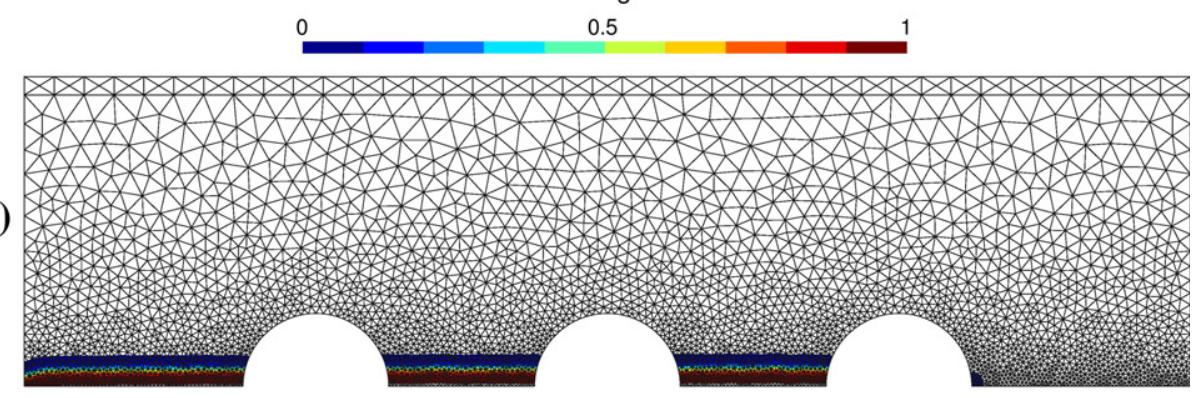

damage

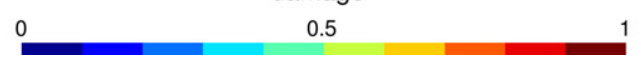

Fig. 19. Evolution of the damage field with the underlying computational mesh. 
the symmetry of the geometry. The upper part of the plate presents a Young modulus ten times larger, the imposed load is therefore very close to an imposed displacement during a large part of the computation. This computation illustrates the potential of the method, especially for the initiation of damage and cracks. The computational mesh presents a characteristic element size of about $l_{c} / 10$. Using the arc-tangent damage shape, we obtain a maximum initial damage value $d \ll 0.1$, which means a negligible impact on the global structure stiffness. The Young modulus and Poisson ratio used are respectively $E=36.5 \mathrm{GPa}$ and $v=0.2$, while we choose $l_{c}=4 \times 10^{-2} \mathrm{~m}$ and $Y_{c}=10^{2} \mathrm{~Pa}$.

The damage evolution and the computational mesh are shown in Fig. 19. The corresponding load-displacement curve is depicted in Fig. 18. During the first computation steps, we observe that six small defects have been initiated on the holes, on the horizontal symmetry axis. Then, one after the others, those defects first grow

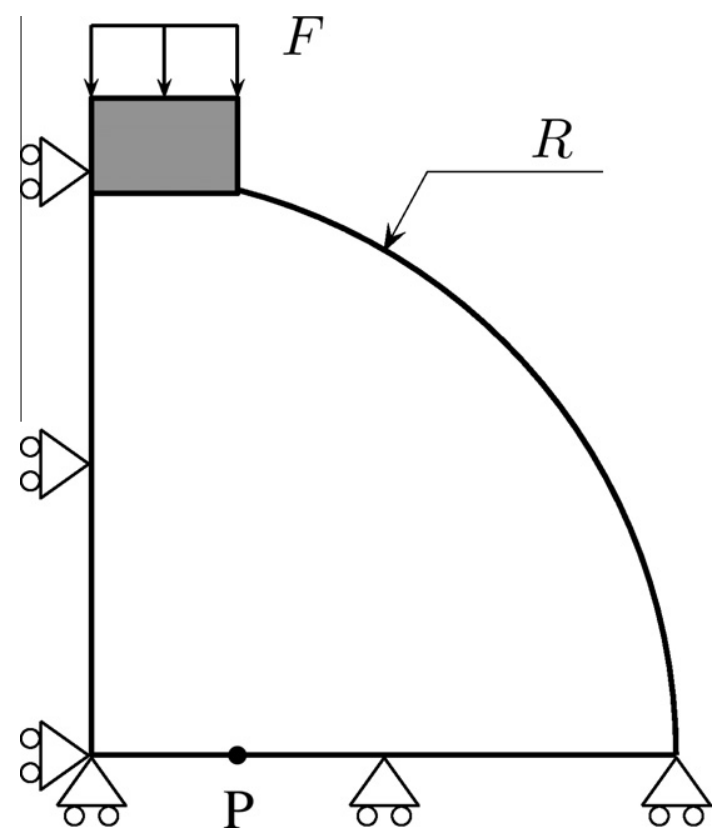

Fig. 20. Domain definition and boundary conditions for the Brazilian test. until reaching a complete damage, and then propagate the crack (fully degraded zone) horizontally. We also observe that the global stiffness of the structure is not much affected as a defect grows from $d=0$ to $d=1$. On the contrary, the crack propagation corresponds to an unstable (strong snapback) process with large loss of stiffness.

This computation took $110 \mathrm{~min}$ for 550 propagation steps on a 7688 triangles mesh using polynomials of order 2 on a single AMD Opteron processor.

\subsection{The Brazilian test}

The loading and boundary conditions of this classical benchmark are depicted in Fig. 20. The specimen thickness is $0.05 \mathrm{~m}$ with a radius of $R=0.1 \mathrm{~m}$ and bearing strips of width $R / 4$ (we take the symmetry of the problem into account, the load $F$ is distributed on a length $R / 8$ ) presenting a Young modulus fifty times larger than the specimen. The physical parameters used are $E=36.5 \mathrm{GPa}$ $v=0.2, Y_{c}=66.6 \mathrm{~Pa}$ and $l_{c}=10^{-2} \mathrm{~m}$. Note that the use of the dissymmetric elastic potential is necessary here to obtain the expected vertical crack propagation.

As for the previous simulation, we check for possible required damage initiations at each time step. The load displacement curves are depicted in Fig. 21, the corresponding displacement fields and iso-zero position in Fig. 22. The results are in very good agreement with observations given in the literature $[28,11]$. Indeed, we observe that defects are initiated at an approximate distance of $2 R /$ 3 from the center $(A)$. The damaged zone grows until a crack appears (B) and propagates downward to the center. This propagation corresponds to a severe snap-back on the load-displacement curve. Once the lower crack tip reaches the center (C), the upper crack tip starts to grow upward (D). The final damage field seems to be in agreement with numerical results from standard non-local models, see Rodríguez-Ferran and Huerta [31] for instance. The convergence of the load-displacement curve, the crack path and the damage initiation process has been observed using several meshes and level set increments $a_{\text {max }}$.

Note finally that damage initiation might have a significant impact on the displacement at some points as point $P$. In a second computation, corresponding to the dashed blue lines of Fig. 21, we considered a single initial damage at the center and did not initiate any other damaged zone during the computation, as done in many crack computations on this benchmark.
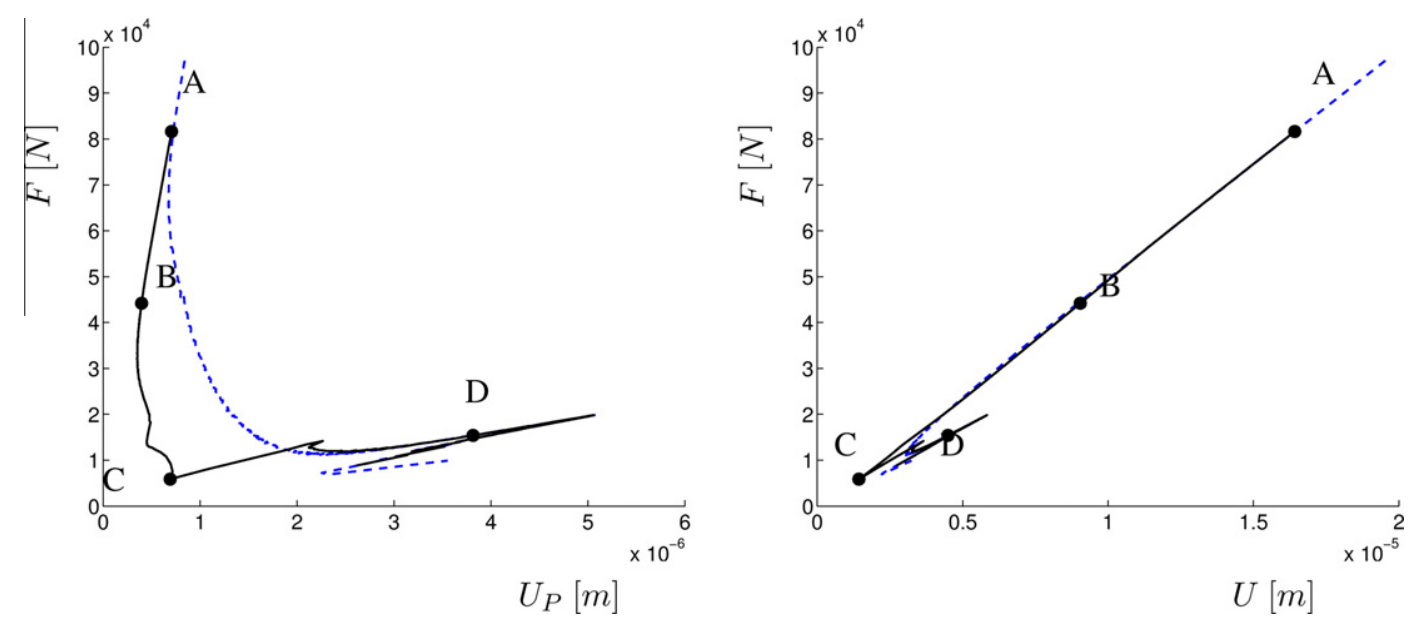

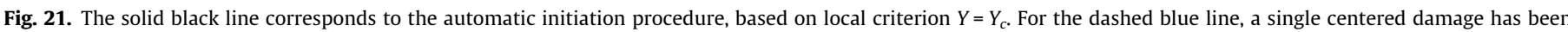

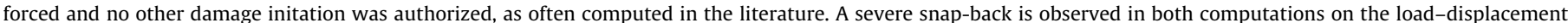

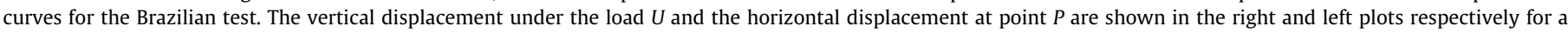
specimen thickness of $0.05 \mathrm{~m}$. 

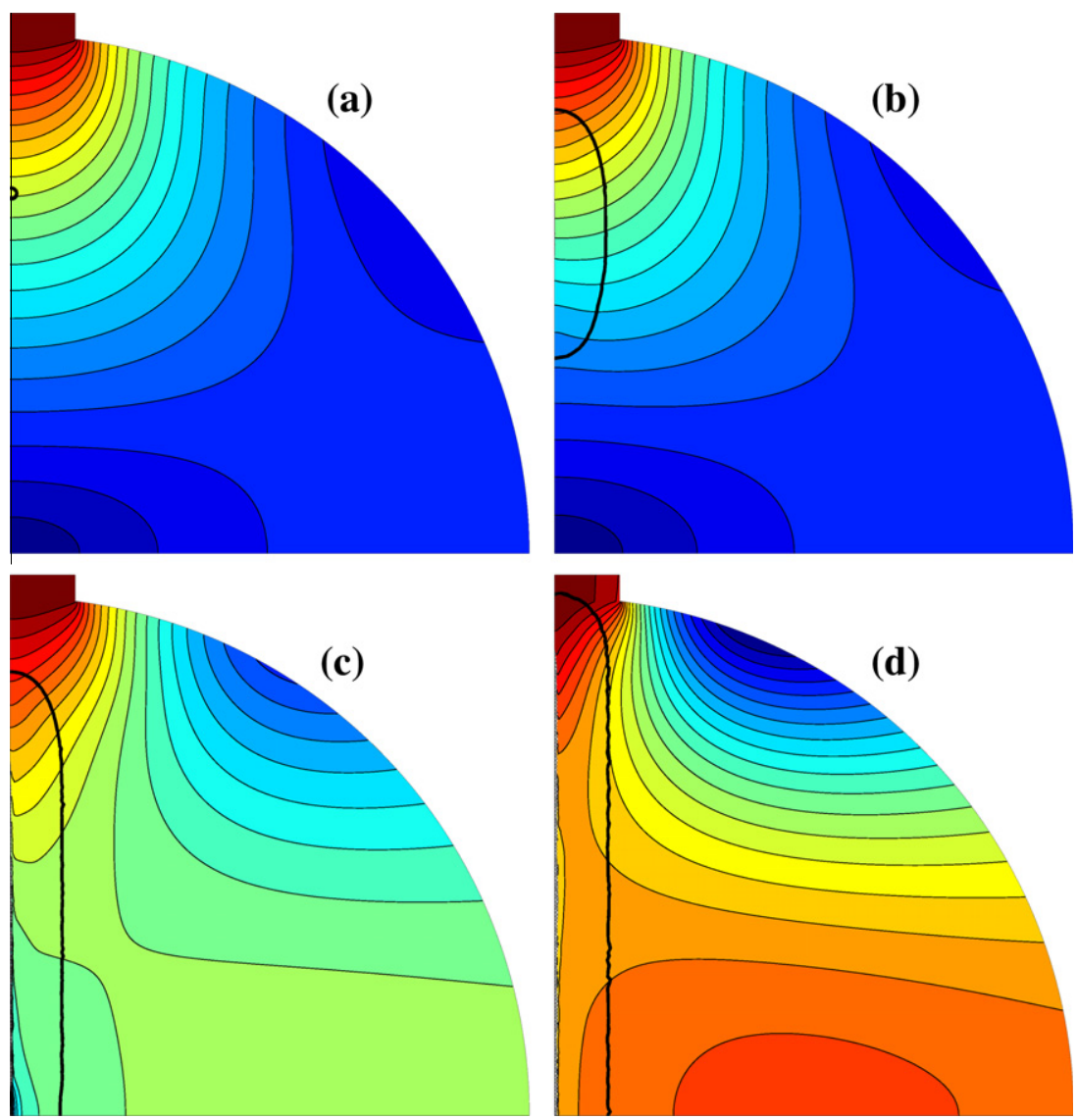

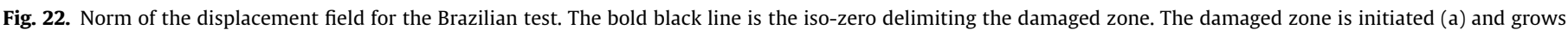
until a crack appears (b) and propagates downward to the center. Once the lower crack tip reaches the center (c), the upper crack tip starts to grow upward (d).

\section{Conclusions}

The TLS approach is an original and promising way to model damage growth. It offers an efficient and consistent non-local damage model with a natural transition to fracture. The damage initiation process is correctly and easily handled since the non-local damage model tends to the local one as the damage tends to zero. We presented here an efficient and simple way to implement this TLS damage model, using a simple variational formulation based on standard finite element discretizations. We also presented an explicit time-independent algorithm in the framework of quasistatic loadings. Finally, we validated the model: the results obtained for classical benchmarks are in good agreement with both the literature and the experiments, even without using any hardening or crack interface model, and the model exhibits good error convergence rates. Less conventional benchmarks were used to demonstrate the potential of the method to initiate defects or to handle interaction of multiple cracks.

Future works will consider more complex three dimensional cases. Although the computational cost associated to the TLS is quite low, there is still plenty of room for costs improvements, especially considering 3D computations. We plan for instance to couple the TLS to standard fracture mechanics and X-FEM in the wake of the advancing frack front.

Another future research topic is the representation of diffuse damage. Indeed, the TLS model is based on the introduction of a characteristic length for the transition zone, between $d=0$ and $d=1$. The present model is therefore not well suited to represent for instance a constant damage in an entire specimen, which may happen without localization phenomena. If the TLS presents a great potential to deal with localization phenomena, a second damage representation should be added to deal with the diffuse pre-localization stage.

Finally, although no explicit cohesive behavior has been introduced in our TLS model, we observed with the L-shaped panel test that cohesive length may appear and automatically vary during the computation. But this cohesive length variation in the TLS model still has to be investigated. Indeed, we observed that the damage profile in function of the level set has a great impact on this possible cohesive behavior, but the link between the two approaches is not clear yet.

\section{Acknowledgments}

The authors acknowledge the french Fondation FNRAE (Fondation Aéronautique et Espace) for their support. Paul-Emile Bernard is a Postdoctoral Researcher supported by the Belgian National Fund for Scientific Research (FNRS).

\section{Appendix A}

\section{A.1. Computational details for the tangent matrix of the elastic computation}

For a given free energy $\varphi(\epsilon)$, the stress tensor reads: $\sigma_{i j}=\frac{\partial \varphi}{\partial \epsilon_{i j}}=\frac{\partial \varphi}{\partial \epsilon_{k}} \frac{\partial \epsilon_{k}}{\partial \epsilon_{i j}}$

with $\epsilon_{i}$ the eigenvalues of the deformation tensor. In the case of the non-linear potential (6), the stress can be written: 
$\sigma_{i j}=L_{k n} \epsilon_{n} \frac{\partial \epsilon_{k}}{\partial \epsilon_{i j}}$

where the matrix:

$L_{k n}=\left.\frac{\partial^{2} \varphi}{\partial \epsilon_{k} \partial \epsilon_{n}}\right|_{\alpha_{i}}$

is considered constant and computed for fixed values of $\alpha_{i}$ based on the previous Newton-Raphson iterate of $\epsilon$.

The derivative of the eigenvalues is computed through the deformation tensor invariants $i_{k}\left(\epsilon_{i j}\right)$ :

$\frac{\partial \epsilon_{n}}{\partial \epsilon_{i j}}=\frac{\partial \epsilon_{n}}{\partial i_{k}} \frac{\partial i_{k}}{\partial \epsilon_{i j}}$.

Eigenvalues $\epsilon_{n}=\epsilon_{n}\left(i_{k}\right)$ are known to be roots of the characteristic polynomial:

$\epsilon^{3}-i_{1} \epsilon^{2}+i_{2} \epsilon-i_{3}=0$.

The analytical expression of the roots, simplified by the assumption of real solutions, can be derived with respect to the invariants. Note that such an analytical computation requires special care to avoid numerical issues as for instance division by zero, appearing in the case of double or triple roots. The tangent matrix then becomes:

$$
\begin{aligned}
H_{i j k l} & =\frac{\partial \sigma_{i j}}{\partial \epsilon_{k l}}=\left.\frac{\partial}{\partial \epsilon_{k l}}\left(\frac{\partial \varphi}{\partial \epsilon_{m}} \frac{\partial \epsilon_{m}}{\partial \epsilon_{i j}}\right) \cong \frac{\partial \sigma_{i j}}{\partial \epsilon_{k l}}\right|_{\alpha_{i}}=\frac{\partial}{\partial \epsilon_{k l}}\left(\epsilon_{m} L_{n m} \frac{\partial \epsilon_{n}}{\partial \epsilon_{i j}}\right) \\
& =\frac{\partial \epsilon_{m}}{\partial \epsilon_{k l}} L_{n m} \frac{\partial \epsilon_{n}}{\partial \epsilon_{i j}}+\epsilon_{m} L_{n m} \frac{\partial^{2} \epsilon_{n}}{\partial \epsilon_{i j} \partial \epsilon_{k l}} .
\end{aligned}
$$

The second derivative in the above expression is eventually computed using invariants:

$\frac{\partial^{2} \epsilon_{n}}{\partial \epsilon_{i j} \partial \epsilon_{k l}}=\frac{\partial}{\partial \epsilon_{k l}}\left(\frac{\partial \epsilon_{n}}{\partial i_{m}} \frac{\partial i_{m}}{\partial \epsilon_{i j}}\right)=\frac{\partial^{2} \epsilon_{n}}{\partial i_{k} \partial i_{p}} \frac{\partial i_{p}}{\partial \epsilon_{k l}} \frac{\partial i_{m}}{\partial \epsilon_{i j}}+\frac{\partial \epsilon_{n}}{\partial i_{m}} \frac{\partial^{2} i_{m}}{\partial \epsilon_{i j} \partial \epsilon_{k l}}$.

\section{A.2. Details about the crack length control algorithm}

Here are some details and explanations about the computation of the parameters $\Delta \mu$ (30) and $k$ (31).

Let us consider that the load factor at time step $n, \mu_{n}$, has been computed using its definition (28). The relationship (29) contains two unknown parameters, $\Delta \mu$ and $k$. First, let us write relationship (29) at time step $n$ for the maximum value of $\bar{Y} / \overline{Y_{c}}$ :

$a_{\max }=k\left(\left(\mu_{n}+|\Delta \mu|\right)^{2}\left(\frac{\widetilde{Y}_{i}}{\overline{Y_{c i}}}\right)_{n, \max }-1\right)=k\left(\left(\mu_{n}+|\Delta \mu|\right)^{2} \frac{1}{\mu_{n}^{2}}-1\right)$.

This relationship provides the value $k(31)$ to obtain a maximum front advance of $a_{\text {max }}$. The parameter $|\Delta \mu|$ still has to be determined. Clearly, we observe in (31) that $k \rightarrow \infty$ for $|\Delta \mu| \rightarrow 0$ and $k \rightarrow 0$ for $|\Delta \mu| \rightarrow \infty$. The former case leads to the activation of only one mode, while the latter leads to move all parts of the front. Both solutions are clearly wrong, the value $\Delta \mu$ must be consistent with the explicit increment $a_{\text {max }}$. In this framework of an explicit method, we simply choose to use the previous load increment, $\Delta \mu_{n}^{n-1}$, as predictor for the value of $\Delta \mu$. Convergence has not been rigorously demonstrated. However, expected convergence rates with the "damage length control" parameter $a_{\max }$ were obtained for the benchmarks considered in this paper.

\section{References}

[1] E. Aifantis, On the structural origin of certain inelastic models, J. Engrg. Mater. Technol. 106 (1984) 326-330.

[2] Z. Bazant, T. Belytschko, T. Chang, Continuum theory for strain-softening, J. Engrg. Mech. 110 (1984) 1666-1692.
[3] S. Bordas, P.V. Nguyen, C. Dunant, A. Guidoum, H. Nguyen-Dang, An extended finite element library, Int. J. Numer. Methods Engrg. 71 (6) (2007) 703-732.

[4] E. Budyn, G. Zi, N. Moës, T. Belytschko, A model for multiple crack growth in brittle materials without remeshing, Int. J. Numer. Methods Engrg. 61 (2004) 1741-1770.

[5] C. Comi, S. Mariani, U. Perego, An extended fe strategy for transition from continuum damage to mode I cohesive crack propagation, Int. J. Numer. Anal. Methods Geomech. 31 (2007) 213-238.

[6] T. Elguedj, A. Gravouil, A. Combescure, Appropriate extended functions for XFEM simulation of plastic fracture mechanics, Comput. Methods Appl. Mech. Engrg. 195 (7-8) (2006) 501-515.

[7] C. Feist, W. Kerber, H. Lehar, G. Hofstetter, A comparative study of numerical models for concrete cracking, in: P. Neittaanmäki, T. Rossi, S. Korotov, E. Onate, J. Périaux, D. Knörzer, (Eds.), Proceedings of ECCOMAS 2004, European Congress on Computational Methods in Applied Sciences and Engineering, Jyväskylä, Finland, 2004.

[8] M. Fremond, B. Nedjar, Damage, gradient of damage and principle of virtual power, Int. J. Solids Struct. 3-8 (1996) 1083-1103.

[9] V. Hakim, A. Karma, Crack path prediction in anisotropic brittle materials Phys. Rev. Lett. 95 (2005) 235501

[10] V. Hakim, A. Karma, Laws of crack motion and phase-field models of fracture, J. Mech. Phys. Solids 57 (2) (2009) 342-368.

[11] D.J. Hannant, K.J. Buckley, J. Croft, The effect of aggregate size on the use of the cylinder splitting test as a measure of tensile strength, Mater. Struct. 6 (1) (1973) 15-21.

[12] P. Jäger, P. Steinmann, E. Kuhl, Modeling three-dimensional crack propagation - a comparison of crack path tracking strategies, Int. J. Numer. Methods Engrg. 76 (9) (2008) 1328-1352.

[13] M. Jirásek, T. Zimmermann, Embedded crack model. Part II: combination with smeared cracks, Int. J. Numer. Methods Engrg. 50 (6) (2001) 12911305.

[14] L. Kachanov, Time of rupture process under creep conditions, Izv. Akad. Nauk USSR Otd. Tekh. Nauk. 8 (1958) 26-31.

[15] A. Karma, D. Kessler, H. Levine, Phase field of mode III dynamic fracture, Phys Rev. Lett. 8704 (2001) 045501

[16] J. Lemaitre, A Course on Damage Mechanics, Springer, 1992.

[17] J. Lemaitre, R. Desmorat, Engineering Damage Mechanics: Ductile, Creep Fatigue and Brittle Failures, Springer-Verlag, Berlin, 2005.

[18] E. Marchandise, P. Geuzaine, N. Chevaugeon, J.F. Remacle, A stabilized finite element method using a discontinuous level set approach for the computation of bubble dynamics, J. Comput. Phys. 225 (2007) 949-974.

[19] J. Mazars, A description of micro- and macroscale damage of concrete structures, Engrg. Fract. Mech. 25 (5-6) (1986) 729-737.

[20] J. Mazars, G. Pijaudier-Cabot, From damage to fracture mechanics and conversely: a combined approach, Int. J. Solids Struct. 33 (20-22) (1996) 3327-3342.

[21] G. Meschke, P. Dumstorff, Energy-based modeling of cohesive and cohesionless cracks via X-FEM, Comput. Methods Appl. Mech. Engrg. 196 (21-24) (2007) 2338-2357.

[22] N. Moës, M. Cloirec, P. Cartraud, J.F. Remacle, A computational approach to handle complex microstructure geometries, Comput. Methods Appl. Mech. Engrg. 192 (28-30) (2003) 3163-3177.

[23] N. Moës, J. Dolbow, T. Belytschko, A finite element method for crack growth without remeshing, Int. J. Numer. Methods Engrg. 46 (1) (1999) 131-150.

[24] N. Moës, C. Stolz, P.E. Bernard, N. Chevaugeon, A level set based model for damage growth: the thick level set approach, Int. J. Numer. Methods Engrg. 86 (2011) 358-380

[25] Q.S. Nguyen, S. Andrieux, The non local generalized standard approach: a consistent gradient theory, C. R. Acad. Sci. Paris 333 (2005) 139-145.

[26] S. Osher, J.A. Sethian, Fronts propagating with curvature dependent speed: algorithms based on Hamilton-Jacobi formulations, J. Comput. Phys. 79 (1988) 12-49.

[27] R. Peerlings, M. Geers, R. de Borst, W. Brekelmans, A critical comparison of non local and gradient-enhanced softening continua, Int. J. Solids Struct. 38 (2001) 7723-7746.

[28] H.J. Petroski, R.P. Ojdrovic, The concrete cylinder: stress analysis and failure modes, Int. J. Fract. 34 (1987) 263-279.

[29] G. Pijaudier-Cabot, Z. Bazant, Non local damage theory, J. Engrg. Mech. 113 (1987) 1512-1533.

[30] G. Pijaudier-Cabot, N. Burlion, Damage and localisation in elastic materials with voids, Mech. Cohes. Frict. Mater. 1 (1996) 129-144.

[31] A. Rodríguez-Ferran, A. Huerta, Error estimation and adaptivity for nonlocal damage models, Int. J. Solids Struct. 37 (48-50) (2000) 7501-7528.

[32] K. Saanouni, N. Belamri, P. Autesserre, Finite element simulation of 3d sheet metal guillotining using advanced fully coupled elastoplasticdamage constitutive equations, Finite Elem. Anal. Des. 46 (2010) 535550 .

[33] H. Schreyer, Z. Chen, One dimensional softening with localization, J. Appl Mech. 53 (1986) 791-979.

[34] J.A. Sethian, Level Set Methods and Fast Marching Methods: Evolving Interfaces in Computational Geometry, Fluid Mechanics, Computer Vision, and Materials Sceince, Cambridge University Press, Cambridge, UK 1999. 
[35] A. Simone, G.N. Wells, L.J. Sluys, From continuous to discontinuous failure in a gradient-enhanced continuum damage model, Comput. Methods Appl. Mech. Engrg. 192 (41-42) (2003) 4581-4607.

[36] N. Sukumar, D. Chopp, B. Moran, Extended finite element method and fast marching method for three-dimensional fatigue crack propagation, Engrg. Fract. Mech. 70 (2003) 29-48.

[37] N. Triantafyllidis, E. Aifantis, A gradient approach to localization of deformation: I hyperelastic model, J. Elasticity 16 (1986) 225237.
[38] J.F. Unger, S. Eckardt, C. Könke, Modelling of cohesive crack growth in concrete structures with the extended finite element method, Comput. Methods Appl. Mech. Engrg. 196 (41-44) (2007) 4087-4100.

[39] B.J. Winkler, Traglastuntersuchungen von unbewehrten und bewehrten betonstrukturen auf der grundlage eines objektiven werkstoffgesetzes für beton, Ph.D. Thesis, Universität Innsbruck, 2001.

[40] E. Wyart, D. Coulon, M. Duflot, T. Pardoen, J.-F. Remacle, F. Lani, A substructured FE-shell/XFE-3D method for crack analysis in thin-walled structures, Int. J. Numer. Methods Engrg. 72 (7) (2007) 757-779. 